\title{
The Absolute Anabelian Geometry of Hyperbolic Curves
}

\author{
SHINICHI MochizUKI
}

\section{Contents:}

$\S 0$. Notations and Conventions

$\S 1$. Review of Anabelian Geometry

$\S 1.1$. The Anabelian Geometry of Number Fields

$\S 1.2$. The Anabelian Geometry of $p$-adic Local Fields

$\S 1.3$. The Anabelian Geometry of Hyperbolic Curves

$\S 2$. Reconstruction of the Logarithmic Special Fiber

Appendix: Terminology of Graph Theory

\section{Introduction}

Let $X_{K}$ be a hyperbolic curve (cf. $\S 0$ below) over a field $K$ of characteristic 0 . Denote its algebraic fundamental group by $\Pi_{X_{K}}$. Thus, we have a natural surjection

$$
\Pi_{X_{K}} \rightarrow G_{K}
$$

of $\Pi_{X_{K}}$ onto the absolute Galois group $G_{K}$ of $K$.

When $K$ is a finite extension of $\mathbb{Q}$ or $\mathbb{Q}_{p}$, and one holds $G_{K}$ fixed, then it is known (cf. [Tama1], [Mzk6]; Theorem 1.3.4 of the present manuscript) that one may recover the curve $X_{K}$ in a functorial fashion from $\Pi_{X_{K}}$. This sort of result may be thought of as a "relative result" (i.e., over $G_{K}$ ). Then the question naturally arises:

To what extent are the "absolute analogues" of this result valid - i.e., what if one does not hold $G_{K}$ fixed?

If $K$ is a number field, then it is still possible to recover $X_{K}$ from $\Pi_{X_{K}}$ (cf. Corollary 1.3.5), by applying the theorem of Neukirch-Uchida (cf. Theorem 1.1.3). On the other 
hand, when $K$ is a $p$-adic local field (i.e., a finite extension of $\mathbb{Q}_{p}$ ), the analogue of the theorem of Neukirch-Uchida fails to hold, and indeed, it is the opinion of the author at the time of writing that it is unlikely (in the $p$-adic local case) that one can recover $X_{K}$ in general (i.e., in the fashion of Corollary 1.3.5) from $\Pi_{X_{K}}$.

In the present manuscript, we begin by reviewing/surveying in $\S 1$ the anabelian geometry of number fields, $p$-adic local fields, and hyperbolic curves from the point of view of the goal of understanding to what extent the anabelian geometry of hyperbolic curves over p-adic local fields can be made "absolute". Our main result (Theorem 2.7 ), given in $\S 2$, states that when $K$ is a $p$-adic local field, (although we may be unable to recover $X_{K}$ itself) one may recover (in a functorial fashion) the special fiber of $X_{K}$, together with its natural log structure, in an absolute fashion, i.e., solely from the isomorphism class of the profinite group $\Pi_{X_{K}}$.

Acknowledgements: I would like to thank A. Tamagawa for the time that he so generously shared with me in numerous stimulating discussions, and especially for the following: (i) informing me of the arguments used to prove Lemma 1.1.4 in §1.1; (ii) explaining to me the utility of a theorem of Raynaud in the context of $\S 2$ (cf. Lemma 2.4). Also, I would like to thank $F$. Oort, as well as the referee, for various useful remarks.

\section{Section 0: Notations and Conventions}

\section{Numbers:}

We will denote by $\mathbb{N}$ the set of natural numbers, by which we mean the set of integers $n \geq 0$. A number field is defined to be a finite extension of the field of rational numbers $\mathbb{Q}$.

\section{Topological Groups:}

Let $G$ be a Hausdorff topological group, and $H \subseteq G$ a closed subgroup. Let us write

$$
Z_{G}(H) \stackrel{\text { def }}{=}\{g \in G \mid g \cdot h=h \cdot g, \forall h \in H\}
$$

for the centralizer of $H$ in $G$;

$$
N_{G}(H) \stackrel{\text { def }}{=}\left\{g \in G \mid g \cdot H \cdot g^{-1}=H\right\}
$$

for the normalizer of $H$ in $G$; and

$$
C_{G}(H) \stackrel{\text { def }}{=}\left\{g \in G \mid\left(g \cdot H \cdot g^{-1}\right) \bigcap H \text { has finite index in } H, g \cdot H \cdot g^{-1}\right\}
$$


for the commensurator of $H$ in $G$. Note that: (i) $Z_{G}(H), N_{G}(H)$ and $C_{G}(H)$ are subgroups of $G$; (ii) we have inclusions

$$
H, Z_{G}(H) \subseteq N_{G}(H) \subseteq C_{G}(H)
$$

and (iii) $H$ is normal in $N_{G}(H)$.

Note that $Z_{G}(H), N_{G}(H)$ are always closed in $G$, while $C_{G}(H)$ is not necessarily closed in $G$. Indeed, one may construct such an example as follows: Let

$$
M \stackrel{\text { def }}{=} \prod_{\mathbb{N}} \mathbb{Z}_{p}
$$

endowed with the product topology (of the various copies of $\mathbb{Z}_{p}$ equipped with their usual topology). Thus, $M$ is a Hausdorff topological group. For $n \in \mathbb{N}$, write $F^{n}(M) \subseteq M$ for the sub-topological group given by the product of the copies of $\mathbb{Z}_{p}$ indexed by $m \geq n$. Write $\operatorname{Aut}_{F}(M)$ for the set of automorphisms of the topological group $M$ that preserve the filtration $F^{*}(M)$ on $M$. If $\alpha \in \operatorname{Aut}_{F}(M)$, then for every $n \in \mathbb{N}$, $\alpha$ induces a continuous homomorphism $\alpha_{n}: M / F^{n}(M) \rightarrow M / F^{n}(M)$ which is clearly surjective, hence an isomorphism (since $M / F^{n}(M)$ is profinite and topologically finitely generated - cf. [FJ], Proposition 15.3). It thus follows that $\alpha$ induces an isomorphism $F^{n}(M) \stackrel{\sim}{\rightarrow} F^{n}(M)$, hence that the inverse of $\alpha$ also lies in $\operatorname{Aut}_{F}(M)$. In particular, we conclude that $\operatorname{Aut}_{F}(M)$ is a group. Equip $\operatorname{Aut}_{F}(M)$ with the coarsest topology for which all of the homomorphisms $\operatorname{Aut}_{F}(M) \rightarrow \operatorname{Aut}\left(M / F^{n}(M)\right)\left(\right.$ where $\operatorname{Aut}\left(M / F^{n}(M)\right) \cong$ $G L_{n}\left(\mathbb{Z}_{p}\right)$ is equipped with its usual topology) are continuous. Note that relative to this topology, $\operatorname{Aut}_{F}(M)$ forms a Hausdorff topological group. Now define $G$ to be the semi-direct product of $M$ with $\operatorname{Aut}_{F}(M)$ (so $G$ is a Hausdorff topological group), and $H$ to be

$$
\prod_{n \in \mathbb{N}} p^{n} \cdot \mathbb{Z}_{p} \subseteq \prod_{\mathbb{N}} \mathbb{Z}_{p}=M
$$

(so $H \subseteq G$ is a closed subgroup). Then $C_{G}(H)$ is not closed in $G$. For instance, if one denotes by $e_{n} \in \prod_{\mathbb{N}} \mathbb{Z}_{p}$ the vector with a 1 in the $n$-th place and zeroes elsewhere, then the limit $A_{\infty}$ (where

$$
A_{\infty}\left(e_{n}\right) \stackrel{\text { def }}{=} e_{n}+e_{n+1}
$$

for all $n \in \mathbb{N}$ ) of the automorphisms $A_{m} \in C_{G}(H)$ (where $A_{m}\left(e_{n}\right) \stackrel{\text { def }}{=} e_{n}+e_{n+1}$ if $n \leq m$, $A_{m}\left(e_{n}\right) \stackrel{\text { def }}{=} e_{n}$ if $\left.n>m\right)$ is not contained in $C_{G}(H)$.

\section{Definition 0.1.}

(i) Let $G$ be a profinite group. Then we shall say that $G$ is slim if the centralizer $Z_{G}(H)$ of any open subgroup $H \subseteq G$ in $G$ is trivial. 
(ii) We shall say that a continuous homomorphism of profinite groups $G \rightarrow H$ is relatively slim if the centralizer in $H$ of the image of every open subgroup of $G$ is trivial.

(iii) We shall say that a closed subgroup $H \subseteq G$ of a profinite group $G$ is commensurably (respectively, normally) terminal if the commensurator $C_{G}(H)$ (respectively, normalizer $\left.N_{G}(H)\right)$ is equal to $H$.

Remark 0.1.1. Thus, a profinite group $G$ is slim if and only if the identity morphism $G \rightarrow G$ is relatively slim. Moreover, if $H \subseteq G$ is a closed subgroup such that the inclusion $H \hookrightarrow G$ is relatively slim, then both $H$ and $G$ are slim.

Remark 0.1.2. It is a formal consequence of the definitions that:

$$
\text { commensurably terminal } \Longrightarrow \text { normally terminal }
$$

and that (if $H \subseteq G$ is a closed subgroup of a profinite group $G$, then):

$$
H \subseteq G \text { commensurably terminal, } H \text { slim } \Longrightarrow
$$

the inclusion $H \hookrightarrow G$ is relatively slim

Remark 0.1.3. It was pointed out to the author by F. Oort that a profinite group is slim if and only if every open subgroup of $G$ has trivial center. (Indeed, the necessity of this condition is clear. Its sufficiency may be shown as follows: If $H \subseteq G$ is an open subgroup, then for any $h \in Z_{G}(H)$, let $H^{\prime} \subseteq G$ by the (necessarily open) subgroup generated by $H$ and $h$. Thus, $h$ lies in the center of $H^{\prime}$, which is trivial by assumption.) This property of a profinite group - i.e., that every open subgroup be center-free was investigated in detail in [Naka1](cf., e.g., Corollaries 1.3.3, 1.3.4).

\section{Curves:}

Suppose that $g \geq 0$ is an integer. Then a family of curves of genus $g$

$$
X \rightarrow S
$$

is defined to be a smooth, proper, geometrically connected morphism $X \rightarrow S$ whose geometric fibers are curves of genus $g$.

Suppose that $g, r \geq 0$ are integers such that $2 g-2+r>0$. We shall denote the moduli stack of r-pointed stable curves of genus $g$ (where we assume the points to be unordered) by $\overline{\mathcal{M}}_{g, r}$ (cf. [DM], [Knud] for an exposition of the theory of such curves; strictly speaking, [Knud] treats the finite étale covering of $\overline{\mathcal{M}}_{g, r}$ determined by ordering 
the marked points). The open substack $\mathcal{M}_{g, r} \subseteq \overline{\mathcal{M}}_{g, r}$ of smooth curves will be referred to as the moduli stack of smooth r-pointed stable curves of genus $g$ or, alternatively, as the moduli stack of hyperbolic curves of type $(g, r)$.

A family of hyperbolic curves of type $(g, r)$

$$
X \rightarrow S
$$

is defined to be a morphism which factors $X \hookrightarrow Y \rightarrow S$ as the composite of an open immersion $X \hookrightarrow Y$ onto the complement $Y \backslash D$ of a relative divisor $D \subseteq Y$ which is finite étale over $S$ of relative degree $r$, and a family $Y \rightarrow S$ of curves of genus $g$. One checks easily that, if $S$ is normal, then the pair $(Y, D)$ is unique up to canonical isomorphism. (Indeed, when $S$ is the spectrum of a field, this fact is well-known from the elementary theory of algebraic curves. Next, we consider an arbitrary connected normal $S$ on which a prime $l$ is invertible (which, by Zariski localization, we may assume without loss of generality). Denote by $S^{\prime} \rightarrow S$ the finite étale covering parametrizing orderings of the marked points and trivializations of the l-torsion points of the Jacobian of $Y$. Note that $S^{\prime} \rightarrow S$ is independent of the choice of $(Y, D)$, since (by the normality of $S$ ), $S^{\prime}$ may be constructed as the normalization of $S$ in the function field of $S^{\prime}$ (which is independent of the choice of $(Y, D)$ since the restriction of $(Y, D)$ to the generic point of $S$ has already been shown to be unique). Thus, the uniqueness of $(Y, D)$ follows by considering the classifying morphism (associated to $(Y, D)$ ) from $S^{\prime}$ to the finite étale covering of $\left(\mathcal{M}_{g, r}\right)_{\mathbb{Z}\left[\frac{1}{\tau}\right]}$ parametrizing orderings of the marked points and trivializations of the $l$ torsion points of the Jacobian [since this covering is well-known to be a scheme, for $l$ sufficiently large].) We shall refer to $Y$ (respectively, $D ; D ; D$ ) as the compactification (respectively, divisor at infinity; divisor of cusps; divisor of marked points) of $X$. A family of hyperbolic curves $X \rightarrow S$ is defined to be a morphism $X \rightarrow S$ such that the restriction of this morphism to each connected component of $S$ is a family of hyperbolic curves of type $(g, r)$ for some integers $(g, r)$ as above.

\section{Section 1: Review of Anabelian Geometry}

\section{$\S 1.1$. The Anabelian Geometry of Number Fields}

In this $\S$, we review well-known anabelian (and related) properties of the Galois groups of number fields and (mainly p-adic) local fields.

Let $F$ be a number field. Fix an algebraic closure $\bar{F}$ of $F$ and denote the resulting absolute Galois group of $F$ by $G_{F}$. Let $\mathfrak{p}$ be a (not necessarily nonarchimedean!) prime of $F$. Write $G_{\mathfrak{p}} \subseteq G_{F}$ for the decomposition group (well-defined up to conjugacy) 
associated to $\mathfrak{p}$ and $F_{\mathfrak{p}}$ for the completion of $F$ at $\mathfrak{p}$. Then we have the following result (cf. also Corollary 1.3.3 below for a generalization of the slimness of $G_{\mathfrak{p}}, G_{F}$ ):

Theorem 1.1.1. (Slimness and Commensurable Terminality) Suppose that $\mathfrak{p}$ is nonarchimedean. Then:

(i) The closed subgroup $G_{\mathfrak{p}} \subseteq G_{F}$ is commensurably terminal.

(ii) The inclusion $G_{\mathfrak{p}} \hookrightarrow G_{F}$ is relatively slim. In particular, $G_{\mathfrak{p}}, G_{F}$ are slim.

Proof. Assertion (i) is a formal consequence of [NSW], Corollary 12.1.3. As for assertion (ii), the slimness of $G_{\mathfrak{p}}$ follows from local class field theory (cf., e.g., [Serre2]). (That is, if $\sigma \in G_{\mathfrak{p}}$ commutes with an open subgroup $H \subseteq G_{\mathfrak{p}}$, then $\sigma$ induces the trivial action on the abelianization $H^{\mathrm{ab}}$. But, by local class field theory, $H^{\mathrm{ab}}$ may be identified with the profinite completion of $K^{\times}$, where $K$ is the finite extension of $F_{\mathfrak{p}}$ determined by $H$. Thus, $\sigma$ acts trivially on all sufficiently large finite extensions $K$ of $F_{\mathfrak{p}}$, so $\sigma=1$, as desired.) Relative slimness thus follows formally from the slimness of $G_{\mathfrak{p}}$ and (i) (cf. Remark 0.1.2).

Theorem 1.1.2. (Topologically Finitely Generated Closed Normal Subgroups) Every topologically finitely generated closed normal subgroup of $G_{F}$ is trivial.

Proof. This follows from [FJ], Theorem 15.10.

Theorem 1.1.3. (The Neukirch-Uchida Theorem on the Anabelian Nature of Number Fields) Let $F_{1}, F_{2}$ be number fields. Let $\bar{F}_{1}$ (respectively, $\bar{F}_{2}$ ) be an algebraic closure of $F_{1}$ (respectively, $\left.F_{2}\right)$. Write $\operatorname{Isom}\left(\bar{F}_{2} / F_{2}, \bar{F}_{1} / F_{1}\right)$ for the set of field isomorphisms $\bar{F}_{2} \stackrel{\sim}{\rightarrow} \bar{F}_{1}$ that map $F_{2}$ onto $F_{1}$. Then the natural map

$$
\operatorname{Isom}\left(\bar{F}_{2} / F_{2}, \bar{F}_{1} / F_{1}\right) \rightarrow \operatorname{Isom}\left(\operatorname{Gal}\left(\bar{F}_{1} / F_{1}\right), \operatorname{Gal}\left(\bar{F}_{2} / F_{2}\right)\right)
$$

is bijective.

Proof. This is the content of [NSW], Theorem 12.2.1.

Remark 1.1.3.1. It is important to note, however, that the analogue of Theorem 1.1.3 for finite extensions of $\mathbb{Q}_{p}$ is false (cf. [NSW], p. 674). Nevertheless, by considering isomorphisms of Galois groups that preserve the higher ramification filtration, one may obtain a partial analogue of this theorem for p-adic local fields (cf. [Mzk5]). 
Next, we would like to consider a situation that arises frequently in anabelian geometry. Suppose that $G$ is equal to $G_{F}$ or $G_{\mathfrak{p}}$ (where we assume now that $\mathfrak{p}$ is nonarchimedean!), and that we are given an exact sequence of profinite groups:

$$
1 \rightarrow \Delta \rightarrow \Pi \rightarrow G \rightarrow 1
$$

Suppose, moreover, that this sequence splits over some open subgroup of $G$, and that $\Delta$ is topologically finitely generated. The following result was related to the author by $A$. Tamagawa:

\section{Lemma 1.1.4. (Intrinsic Characterization of Arithmetic Quotients)}

(i) Suppose that $G=G_{F}$. Let $\Pi^{\prime} \subseteq \Pi$ be an open subgroup. Then the kernel of the homomorphism $\Pi^{\prime} \rightarrow G$ may be characterized as the unique maximal closed normal subgroup of $\Pi^{\prime}$ which is topologically finitely generated.

(ii) Suppose that $G=G_{\mathfrak{p}}$. Assume further that for every open subgroup $\Pi^{\prime \prime} \subseteq \Pi$, the abelianization $\left(\Delta^{\prime \prime}\right)$ ab of $\Delta^{\prime \prime}\left(\right.$ where $\left.\Delta^{\prime \prime} \stackrel{\text { def }}{=} \Pi^{\prime \prime} \cap \Delta\right)$ satisfies the following property:

(*) The maximal torsion-free quotient $\left(\Delta^{\prime \prime}\right)^{\mathrm{ab}} \rightarrow Q^{\prime \prime}$ of $\left(\Delta^{\prime \prime}\right)^{\mathrm{ab}}$ on which the action of $G^{\prime \prime} \stackrel{\text { def }}{=} \Pi^{\prime \prime} / \Delta^{\prime \prime}$ (by conjugation) is trivial is a finitely generated free $\widehat{\mathbb{Z}}$-module.

Let $\Pi^{\prime} \subseteq \Pi$ be an arbitrary open subgroup. Then:

$$
\left[G: G^{\prime}\right] \cdot\left[F_{\mathfrak{p}}: \mathbb{Q}_{p}\right]=\operatorname{dim}_{\mathbb{Q}_{p}}\left(\left(\Pi^{\prime}\right)^{\mathrm{ab}} \otimes_{\widehat{\mathbb{Z}}} \mathbb{Q}_{p}\right)-\operatorname{dim}_{\mathbb{Q}_{l}}\left(\left(\Pi^{\prime}\right)^{\mathrm{ab}} \otimes_{\widehat{\mathbb{Z}}} \mathbb{Q}_{l}\right)
$$

(where $\Delta^{\prime} \stackrel{\text { def }}{=} \Delta \bigcap \Pi^{\prime} ; G^{\prime} \stackrel{\text { def }}{=} \Pi^{\prime} / \Delta^{\prime} ; p$ is the rational prime that $\mathfrak{p}$ divides; and $l$ is any prime number distinct from $p$ ). (In fact, $p$ may also be characterized as the unique prime number for which the difference on the right is nonzero for infinitely many prime numbers l.) In particular, the subgroup $\Delta \subseteq \Pi$ may be characterized as the intersection of those open subgroups $\Pi^{\prime} \subseteq \Pi$ such that:

$$
\left[G: G^{\prime}\right]=\left[\Pi: \Pi^{\prime}\right]
$$

(i.e., such that $\left[G: G^{\prime}\right] \cdot\left[F_{\mathfrak{p}}: \mathbb{Q}_{p}\right]=\left[\Pi: \Pi^{\prime}\right] \cdot\left([G: G] \cdot\left[F_{\mathfrak{p}}: \mathbb{Q}_{p}\right]\right)$ ).

Proof. Assertion (i) is a formal consequence of Theorem 1.1.2.

Now we turn to assertion (ii). Denote by $K^{\prime}$ the finite extension of $F_{\mathfrak{p}}$ determined by $G^{\prime}$. Then:

$$
\left[G: G^{\prime}\right] \cdot\left[F_{\mathfrak{p}}: \mathbb{Q}_{p}\right]=\left[K^{\prime}: \mathbb{Q}_{p}\right]
$$


Next, let us observe that we have an exact sequence

$$
0 \rightarrow \operatorname{Im}\left(\Delta^{\prime}\right) \rightarrow\left(\Pi^{\prime}\right)^{\mathrm{ab}} \rightarrow\left(G^{\prime}\right)^{\mathrm{ab}} \rightarrow 0
$$

(where $\operatorname{Im}\left(\Delta^{\prime}\right)$ is the image of $\Delta^{\prime}$ in $\left(\Pi^{\prime}\right)^{\text {ab }}$ ) of finitely generated $\widehat{\mathbb{Z}}$-modules. Note, moreover, that we have a natural surjection $Q^{\prime} \rightarrow \operatorname{Im}\left(\Delta^{\prime}\right) /$ (torsion) (where $Q^{\prime}$ is as in $(*))$. On the other hand, it follows formally from our assumption that $\Pi^{\prime} \rightarrow G^{\prime}$ splits over an open subgroup of $G^{\prime}$ (together with the well-known fact that the group cohomology of a finite group is always annihilated by the order to the group) that this natural surjection is, in fact, an isomorphism. In particular, it follows formally from $(*)$ that:

$\operatorname{dim}_{\mathbb{Q}_{p}}\left(\left(\Pi^{\prime}\right)^{\mathrm{ab}} \otimes_{\widehat{\mathbb{Z}}} \mathbb{Q}_{p}\right)-\operatorname{dim}_{\mathbb{Q}_{l}}\left(\left(\Pi^{\prime}\right)^{\mathrm{ab}} \otimes_{\widehat{\mathbb{Z}}} \mathbb{Q}_{l}\right)=\operatorname{dim}_{\mathbb{Q}_{p}}\left(\left(G^{\prime}\right)^{\mathrm{ab}} \otimes_{\widehat{\mathbb{Z}}} \mathbb{Q}_{p}\right)-\operatorname{dim}_{\mathbb{Q}_{l}}\left(\left(G^{\prime}\right)^{\mathrm{ab}} \otimes_{\widehat{\mathbb{Z}}} \mathbb{Q}_{l}\right)$

Thus, to complete the proof of Lemma 1.1.4, it suffices to prove that:

$$
\left[K^{\prime}: \mathbb{Q}_{p}\right]=\operatorname{dim}_{\mathbb{Q}_{p}}\left(\left(G^{\prime}\right)^{\mathrm{ab}} \otimes_{\widehat{\mathbb{Z}}} \mathbb{Q}_{p}\right)-\operatorname{dim}_{\mathbb{Q}_{l}}\left(\left(G^{\prime}\right)^{\mathrm{ab}} \otimes_{\widehat{\mathbb{Z}}} \mathbb{Q}_{l}\right)
$$

But this is a formal consequence of local class field theory (cf., e.g., [Serre2]; $§ 1.2$ below), i.e., the fact that $\left(G^{\prime}\right)^{\mathrm{ab}}$ is isomorphic to the profinite completion of $\left(K^{\prime}\right)^{\times}$.

Typically, in applications involving hyperbolic curves, one shows that the condition $(*)$ of Lemma 1.1.4 is satisfied by applying the following:

Lemma 1.1.5. (Tate Modules of Semi-abelian Varieties) Let $K$ be a finite extension of $\mathbb{Q}_{p}$. Fix an algebraic closure $\bar{K}$ of $K$; write $G_{K} \stackrel{\text { def }}{=} \operatorname{Gal}(\bar{K} / K)$. Let $A$ be a semi-abelian variety over $K$. Denote the resulting (profinite) Tate module of $A$ by:

$$
T(A) \stackrel{\text { def }}{=} \operatorname{Hom}(\mathbb{Q} / \mathbb{Z}, A(\bar{K}))
$$

Then the maximal torsion-free quotient $T(A) \rightarrow Q$ on which $G_{K}$ acts trivially is a finitely generated free $\widehat{\mathbb{Z}}$-module.

Proof. A semi-abelian variety is an extension of an abelian variety by a torus. Thus, $T(A)$ is the extension of the Tate module of an abelian variety by the Tate module of a torus. Moreover, since (after restricting to some open subgroup of $G_{K}$ ) the Tate module of a torus is isomorphic to the direct sum of a finite number of copies of $\widehat{\mathbb{Z}}(1)$, we thus conclude that the image of the Tate module of the torus in $Q$ is necessarily zero. In particular, we may assume for the remainder of the proof without loss of generality that $A$ is an abelian variety. 
Now it follows from the theory of [FC] (cf., in particular, [FC], Chapter III, Corollary 7.3 ), that $T(A)$ fits into exact sequences (of $G_{K}$-modules)

$$
\begin{gathered}
0 \rightarrow T_{\text {good }} \rightarrow T(A) \rightarrow T_{\text {com }} \rightarrow 0 \\
0 \rightarrow T_{\text {trc }} \rightarrow T_{\text {good }} \rightarrow T(B) \rightarrow 0
\end{gathered}
$$

where $T(B)$ is the Tate module of an abelian variety $B$ over $K$ with potentially good reduction; and $T_{\text {com }}=M_{\text {com }} \otimes_{\mathbb{Z}} \widehat{\mathbb{Z}}, T_{\text {trc }}=M_{\text {trc }} \otimes_{\mathbb{Z}} \widehat{\mathbb{Z}}(1)$ for finitely generated free $\mathbb{Z}$ modules $M_{\mathrm{com}}, M_{\mathrm{trc}}$ on which $G_{K}$ acts via a finite quotient. [Here, "com" (respectively, "trc") stands for "combinatorial" (respectively, "toric").] It is thus evident that $T_{\text {trc }}$ maps to 0 in $Q$. Moreover, by [Mzk4], Lemma 8.1 (the proof of which is valid for arbitrary $B$, even though in loc. cit., this result is only stated in the case of a Jacobian), and the Riemann Hypothesis for abelian varieties over finite fields (cf., e.g., [Mumf], p. $206)$, it follows that $T(B)$ also maps to 0 in $Q$. Thus, we conclude that $Q$ is equal to the maximal torsion-free quotient of $T_{\text {com }}$ on which $G_{K}$ acts trivially. Since $\widehat{\mathbb{Z}}$ is $\mathbb{Z}$-flat, however, this implies that $Q$ is equal to the result of applying $\otimes_{\mathbb{Z}} \widehat{\mathbb{Z}}$ to the maximal torsion-free quotient of $M_{\text {com }}$ on which $G_{K}$ acts trivially. But this last quotient is manifestly finitely generated and free over $\mathbb{Z}$. This completes the proof.

\section{$\S 1.2$. The Anabelian Geometry of $p$-adic Local Fields}

In this $\S$, we review certain well-known "group-theoretic" properties of Galois groups of p-adic local fields, i.e., properties preserved by arbitrary isomorphisms between such Galois groups.

For $i=1,2$, let $p_{i}$ be a prime number. Let $K_{i}$ be a finite extension of $\mathbb{Q}_{p_{i}}$. We denote the ring of integers (respectively, maximal ideal; residue field) of $K_{i}$ by $\mathcal{O}_{K_{i}}$ (respectively, $\mathfrak{m}_{K_{i}} ; k_{i}$ ). Also, we assume that we have chosen an algebraic closures $\bar{K}_{i}$ of $K_{i}$ and write

$$
G_{K_{i}} \stackrel{\text { def }}{=} \operatorname{Gal}\left(\bar{K}_{i} / K_{i}\right)
$$

for the corresponding absolute Galois group of $K_{i}$. Thus, by local class field theory (cf., e.g., [Serre2]), we have a natural isomorphism

$$
\left(K_{i}^{\times}\right)^{\wedge} \stackrel{\sim}{\rightarrow} G_{K_{i}}^{\mathrm{ab}}
$$

(where the " $\wedge$ " denotes the profinite completion of an abelian group; " $\times$ " denotes the group of units of a ring; and "ab" denotes the maximal abelian quotient of a topological group). In particular, $G_{K_{i}}^{\mathrm{ab}}$ fits into an exact sequence

$$
0 \rightarrow \mathcal{O}_{K_{i}}^{\times} \rightarrow G_{K_{i}}^{\mathrm{ab}} \rightarrow \widehat{\mathbb{Z}} \rightarrow 0
$$


(arising from a similar exact sequence for $\left.\left(K_{i}^{\times}\right)^{\wedge}\right)$. Moreover, we obtain natural inclusions

$$
\begin{gathered}
k_{i}^{\times} \hookrightarrow \mathcal{O}_{K_{i}}^{\times} \subseteq K_{i}^{\times} \hookrightarrow G_{K_{i}}^{\mathrm{ab}} \\
K_{i}^{\times} / \mathcal{O}_{K_{i}}^{\times} \stackrel{\sim}{\rightarrow} \mathbb{Z} \hookrightarrow G_{K_{i}}^{\mathrm{ab}} / \operatorname{Im}\left(\mathcal{O}_{K_{i}}^{\times}\right)
\end{gathered}
$$

(where " $\stackrel{\sim}{\rightarrow}$ " denotes the morphism induced by the valuation on $K_{i}^{\times}$) by considering the Teichmüller representatives of elements of $k_{i}^{\times}$and the Frobenius element, respectively. Also, in the following discussion we shall write:

$$
\begin{gathered}
\boldsymbol{\mu}_{\widehat{\mathbb{Z}}}\left(\bar{K}_{i}\right) \stackrel{\text { def }}{=} \operatorname{Hom}\left(\mathbb{Q} / \mathbb{Z}, \bar{K}_{i}^{\times}\right) ; \quad \boldsymbol{\mu}_{\widehat{\mathbb{Z}}}\left(\bar{K}_{i}\right) \stackrel{\text { def }}{=} \boldsymbol{\mu}_{\widehat{\mathbb{Z}}}\left(\bar{K}_{i}\right) \otimes_{\widehat{\mathbb{Z}}} \widehat{\mathbb{Z}}^{\prime} ; \\
\boldsymbol{\mu}_{\mathbb{Q} / \mathbb{Z}}\left(\bar{K}_{i}\right) \stackrel{\text { def }}{=} \boldsymbol{\mu}_{\widehat{\mathbb{Z}}}\left(\bar{K}_{i}\right) \otimes_{\widehat{\mathbb{Z}}} \mathbb{Q} / \mathbb{Z}
\end{gathered}
$$

(where $\widehat{\mathbb{Z}}^{\prime} \stackrel{\text { def }}{=} \widehat{\mathbb{Z}} / \mathbb{Z}_{p}$ ). Finally, we denote the cyclotomic character of $G_{K_{i}}$ by:

$$
\chi_{i}: G_{K_{i}} \rightarrow \widehat{\mathbb{Z}}^{\times}
$$

\section{Proposition 1.2.1. (Invariants of Arbitrary Isomorphisms of Galois Groups} of Local Fields) Suppose that we are given an isomorphism of profinite groups:

$$
\alpha: G_{K_{1}} \stackrel{\sim}{\rightarrow} G_{K_{2}}
$$

Then:

(i) We have: $p_{1}=p_{2}$. Thus, (in the remainder of this proposition and its proof) we shall write $p \stackrel{\text { def }}{=} p_{1}=p_{2}$.

(ii) $\alpha$ induces an isomorphism $I_{K_{1}} \stackrel{\sim}{\rightarrow} I_{K_{2}}$ between the respective inertia subgroups of $G_{K_{1}}, G_{K_{2}}$.

(iii) The isomorphism $\alpha^{\mathrm{ab}}: G_{K_{1}}^{\mathrm{ab}} \stackrel{\sim}{\rightarrow} G_{K_{2}}^{\mathrm{ab}}$ induced by $\alpha$ preserves the images $\operatorname{Im}\left(\mathcal{O}_{K_{i}}^{\times}\right), \operatorname{Im}\left(k_{i}^{\times}\right), \operatorname{Im}\left(K_{i}^{\times}\right)$of the natural morphisms discussed above.

(iv) The morphism induced by $\alpha$ between the respective quotients $G_{K_{i}}^{\mathrm{ab}} / \operatorname{Im}\left(\mathcal{O}_{K_{i}}^{\times}\right)$ preserves the respective Frobenius elements.

(v) $\left[K_{1}: \mathbb{Q}_{p}\right]=\left[K_{2}: \mathbb{Q}_{p}\right] ;\left[k_{1}: \mathbb{F}_{p}\right]=\left[k_{2}: \mathbb{F}_{p}\right]$. In particular, the ramification indices of $K_{1}, K_{2}$ over $\mathbb{Q}_{p}$ coincide.

(vi) The morphisms induced by $\alpha$ on the abelianizations of the various open subgroups of the $G_{K_{i}}$ induce an isomorphism

$$
\boldsymbol{\mu}_{\mathbb{Q} / \mathbb{Z}}\left(\bar{K}_{1}\right) \stackrel{\sim}{\rightarrow} \boldsymbol{\mu}_{\mathbb{Q} / \mathbb{Z}}\left(\bar{K}_{2}\right)
$$


which is Galois-equivariant with respect to $\alpha$. In particular, $\alpha$ preserves the cyclotomic characters $\chi_{i}$.

(vii) The morphism $H^{2}\left(K_{1}, \boldsymbol{\mu}_{\mathbb{Q} / \mathbb{Z}}\left(\bar{K}_{1}\right)\right) \stackrel{\sim}{\rightarrow} H^{2}\left(K_{2}, \boldsymbol{\mu}_{\mathbb{Q} / \mathbb{Z}}\left(\bar{K}_{2}\right)\right)$ induced by $\alpha$ (cf. (vi)) preserves the "residue map"

$$
H^{2}\left(K_{i}, \boldsymbol{\mu}_{\mathbb{Q} / \mathbb{Z}}\left(\bar{K}_{i}\right)\right) \stackrel{\sim}{\rightarrow} \mathbb{Q} / \mathbb{Z}
$$

of local class field theory (cf. [Serre2], §1.1).

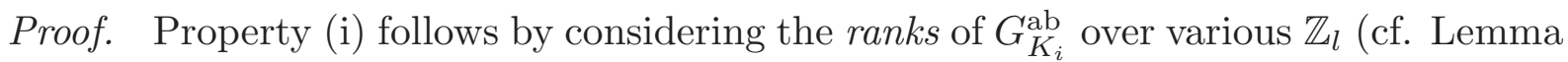
1.1.4, (ii)). Property (iii) for $\operatorname{Im}\left(k_{i}^{\times}\right)$follows from the fact that $\operatorname{Im}\left(k_{i}^{\times}\right)$may be recovered as the prime-to- $p$ torsion subgroup of $G_{K_{i}}^{\mathrm{ab}}$. Property $(\mathrm{v})$ follows for $\left[K_{i}: \mathbb{Q}_{p}\right]$ by considering the $\mathbb{Z}_{p}$-rank of $G_{K_{i}}^{\text {ab }}$ (minus 1 ) and for $\left[k_{i}: \mathbb{F}_{p}\right]$ by considering the cardinality of $\operatorname{Im}\left(k_{i}^{\times}\right)$(plus 1) - cf. (i), (iii). Property (ii) follows from the fact that whether or not a finite extension is unramified may be determined group-theoretically by considering the variation of the ramification index over $\mathbb{Q}_{p}$ (cf. (v)). Property (iii) for $\operatorname{Im}\left(\mathcal{O}_{K_{i}}^{\times}\right)$ follows formally from (ii) (since this image is equal to the image in $G_{K_{i}}^{\mathrm{ab}}$ of $I_{K_{i}}$ ). Property (iv) follows by applying (iii) for $\operatorname{Im}\left(k_{i}^{\times}\right)$to the various open subgroups of $G_{K_{i}}$ that correspond to unramified extensions of $K_{i}$ and using the fact the Frobenius element is the unique element that acts on $\bar{k}_{i}^{\times}$(where $\bar{k}_{i}$ denotes the algebraic closure of $k_{i}$ induced by $\bar{K}_{i}$ ) as multiplication by $\left|k_{1}\right|=\left|k_{2}\right|$ [where, relative to our use of the term "multiplication", we think of the abelian group $\bar{k}_{i}^{\times}$additively]. Here, we note that if $L_{i}$ is a finite extension of $K_{i}$, then the inclusion

$$
G_{K_{i}}^{\mathrm{ab}} \stackrel{\sim}{\rightarrow}\left(K_{i}^{\times}\right)^{\wedge} \hookrightarrow\left(L_{i}^{\times}\right)^{\wedge} \stackrel{\sim}{\rightarrow} G_{L_{i}}^{\mathrm{ab}}
$$

may be reconstructed group-theoretically by considering the Verlagerung, or transfer, map (cf. [Serre2], §2.4). Property (iii) for $\operatorname{Im}\left(K_{i}^{\times}\right)$follows formally from (iv). Property (vi) follows formally from (iii). Finally, property (vii) follows (cf. the theory of the Brauer group of a local field, as exposed, for instance, in [Serre2], §1) from the fact that the morphism $H^{2}\left(K_{i}, \boldsymbol{\mu}_{\mathbb{Q} / \mathbb{Z}}\left(\bar{K}_{i}\right)\right) \stackrel{\sim}{\rightarrow} \mathbb{Q} / \mathbb{Z}$ may be constructed as the composite of the natural isomorphism

$$
H^{2}\left(K_{i}, \boldsymbol{\mu}_{\mathbb{Q} / \mathbb{Z}}\left(\bar{K}_{i}\right)\right)=H^{2}\left(G_{K_{i}}, \boldsymbol{\mu}_{\mathbb{Q} / \mathbb{Z}}\left(\bar{K}_{i}\right)\right) \stackrel{\sim}{\rightarrow} H^{2}\left(G_{K_{i}}, \bar{K}_{i}^{\times}\right)
$$

— which is group-theoretic, by (iii) — with the inverse of the isomorphism

$$
H^{2}\left(\operatorname{Gal}\left(K_{i}^{\mathrm{unr}} / K_{i}\right),\left(K_{i}^{\mathrm{unr}}\right)^{\times}\right) \stackrel{\sim}{\rightarrow} H^{2}\left(G_{K_{i}}, \bar{K}_{i}^{\times}\right)
$$

(where $K_{i}^{\text {unr }}$ denotes the maximal unramified extension of $K_{i}$ ) - which is grouptheoretic, by (ii), (iii) — followed by the natural isomorphism

$$
H^{2}\left(\operatorname{Gal}\left(K_{i}^{\mathrm{unr}} / K_{i}\right),\left(K_{i}^{\mathrm{unr}}\right)^{\times}\right) \stackrel{\sim}{\rightarrow} H^{2}\left(\operatorname{Gal}\left(K_{i}^{\mathrm{unr}} / K_{i}\right), \mathbb{Z}\right)=H^{2}(\widehat{\mathbb{Z}}, \mathbb{Z})=\mathbb{Q} / \mathbb{Z}
$$


— which is group-theoretic, by (ii), (iii), (iv).

Before proceeding, we observe that Proposition 1.2.1, (i), may be extended as follows: Write

$$
\mathfrak{A}_{\mathbb{Q}} \stackrel{\text { def }}{=}|\operatorname{Spec}(\mathbb{Z})| \bigcup\{\infty\}
$$

(where "| - " denotes the underlying set of a scheme) for the set of "all arithmetic primes of $\mathbb{Q} "$. If $v \in \mathfrak{A}_{\mathbb{Q}}$ is equal to $(0) \in|\operatorname{Spec}(\mathbb{Z})|($ respectively, $\infty)$, set $G_{v} \stackrel{\text { def }}{=} G_{\mathbb{Q}}$ (respectively, $G_{v} \stackrel{\text { def }}{=} \operatorname{Gal}(\mathbb{C} / \mathbb{R})$ ). If $v \in|\operatorname{Spec}(\mathbb{Z})| \subseteq \mathfrak{A}_{\mathbb{Q}}$ is equal to the prime determined by a prime number $p$, set $G_{v} \stackrel{\text { def }}{=} G_{\mathbb{Q}_{p}}$.

Proposition 1.2.2. (Intrinsicity of Arithmetic Types) For $i=1,2$, let $v_{i} \in \mathfrak{A}_{\mathbb{Q}}$. Suppose that $H_{i}$ is an open subgroup of $G_{v_{i}}$. Then $H_{1} \cong H_{2}$ implies $v_{1}=v_{2}$.

Proof. Indeed, open subgroups of $G_{\mathbb{Q}}$ may be distinguished by the fact that their abelianizations fail to be topologically finitely generated. (Indeed, consider the abelian extensions arising from adjoining roots of unity.) By contrast, abelianizations of open subgroups of $G_{\mathbb{R}}$ or $G_{\mathbb{Q}_{p}}$ (cf. the above discussion) are topologically finitely generated. Next, open subgroups of $G_{\mathbb{R}}$ may be distinguished from those of any $G_{\mathbb{Q}_{p}}$ by the fact they are finite. The remainder of Proposition 1.2.2 follows from Proposition 1.2.1, (i).

Next, let us write $\operatorname{Spec}\left(\mathcal{O}_{K_{i}}\right)^{\log }$ for the log scheme obtained by equipping the scheme $\operatorname{Spec}\left(\mathcal{O}_{K_{i}}\right)$ with the log structure defined by the divisor $V\left(\mathfrak{m}_{K_{i}}\right)$. Thus, by pulling back this $\log$ structure via the natural morphism $\operatorname{Spec}\left(k_{i}\right) \hookrightarrow \operatorname{Spec}\left(\mathcal{O}_{K_{i}}\right)$, we obtain a $\log$ scheme $\operatorname{Spec}\left(k_{i}\right)^{\log }$, which we denote by

$$
k_{i}^{\log }
$$

for short. Note that the "étale monoid" that defines the log structure on $k_{i}^{\log }$ "admits a global chart" in the sense that it is defined by a single constant monoid (in the Zariski topology of $\left.\operatorname{Spec}\left(k_{i}\right)\right) M_{k_{i}^{\log }}$, which fits into a natural exact sequence (of monoids):

$$
1 \rightarrow k_{i}^{\times} \rightarrow M_{k_{i}^{\log }} \rightarrow \mathbb{N} \rightarrow 0
$$

Thus, the $k_{i}^{\times}$-torsor $U_{i}$ determined by considering the inverse image of $1 \in \mathbb{N}$ in this sequence may be identified with the $k_{i}^{\times}$-torsor of uniformizers $\in \mathfrak{m}_{K_{i}}$ considered modulo $\mathfrak{m}_{K_{i}}^{2}$.

Next, let us write

$$
G_{K_{i}} \rightarrow G_{k_{i}}^{\log }
$$


for the quotient defined by the maximal tamely ramified extension $K_{i}^{\text {tame }}$ of $K_{i}$. Thus, $G_{k_{i}}^{\log }$ may also be thought of as the "logarithmic fundamental group" $\pi_{1}\left(k_{i}^{\log }\right)$ of the log scheme $k_{i}^{\log }$. Moreover, $G_{k_{i}}^{\text {log }}$ fits into a natural exact sequence:

$$
1 \rightarrow \boldsymbol{\mu}_{\widehat{\mathbb{Z}}}\left(\bar{k}_{i}\right) \rightarrow G_{k_{i}}^{\log } \rightarrow \widehat{\mathbb{Z}} \rightarrow 1
$$

where, just as in the case of $K_{i}$, we write:

$$
\begin{gathered}
\boldsymbol{\mu}_{\widehat{\mathbb{Z}}}\left(\bar{k}_{i}\right)=\boldsymbol{\mu}_{\widehat{\mathbb{Z}}^{\prime}}\left(\bar{k}_{i}\right) \stackrel{\text { def }}{=} \operatorname{Hom}\left(\mathbb{Q} / \mathbb{Z}, \bar{k}_{i}^{\times}\right) ; \\
\boldsymbol{\mu}_{\mathbb{Q} / \mathbb{Z}}\left(\bar{k}_{i}\right) \stackrel{\text { def }}{=} \boldsymbol{\mu}_{\widehat{\mathbb{Z}}}\left(\bar{k}_{i}\right) \otimes_{\widehat{\mathbb{Z}}} \mathbb{Q} / \mathbb{Z}
\end{gathered}
$$

The "abelianization" of this exact sequence yields an exact sequence:

$$
1 \rightarrow k_{i}^{\times} \rightarrow\left(G_{k_{i}}^{\text {log }}\right)^{\mathrm{ab}} \rightarrow \widehat{\mathbb{Z}} \rightarrow 1
$$

Now we have the following:

\section{Proposition 1.2.3.}

(i) Any isomorphism $\alpha: G_{K_{1}} \stackrel{\sim}{\rightarrow} G_{K_{2}}$ (as in Proposition 1.2.1) induces an isomor-

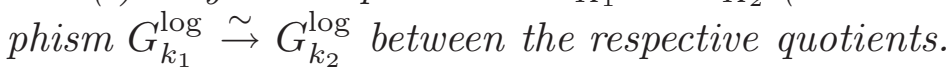

(ii) There is a natural isomorphism of $k_{i}^{\times}$-torsors between the torsor of uniformizers $U_{i}$ discussed above and the $H^{1}\left(\widehat{\mathbb{Z}}, \boldsymbol{\mu}_{\widehat{\mathbb{Z}}}\left(\bar{k}_{i}\right)\right)=k_{i}^{\times}$-torsor $H^{1}\left(G_{k_{i}}^{\log }, \boldsymbol{\mu}_{\widehat{\mathbb{Z}}}\left(\bar{k}_{i}\right)\right)^{[1]}$ of elements of $H^{1}\left(G_{k_{i}}^{\mathrm{log}}, \boldsymbol{\mu}_{\widehat{\mathbb{Z}}}\left(\bar{k}_{i}\right)\right)$ that map to the identity element in $H^{1}\left(\boldsymbol{\mu}_{\widehat{\mathbb{Z}}}\left(\bar{k}_{i}\right), \boldsymbol{\mu}_{\widehat{\mathbb{Z}}}\left(\bar{k}_{i}\right)\right)=$ $\operatorname{Hom}_{\widehat{\mathbb{Z}}^{\prime}}\left(\boldsymbol{\mu}_{\widehat{\mathbb{Z}}}\left(\bar{k}_{i}\right), \boldsymbol{\mu}_{\widehat{\mathbb{Z}}}\left(\bar{k}_{i}\right)\right)$. This isomorphism is defined by associating to a uniformizer $\pi \in U_{i}$ the $\boldsymbol{\mu}_{\widehat{\mathbb{Z}}}\left(\bar{k}_{i}\right)$-torsor over $k_{i}^{\log }$ determined by the roots $\pi^{1 / N}$, as $N$ ranges (multiplicatively) over the positive integers prime to $p_{i}$.

(iii) The profinite group $G_{k_{i}}^{\log }$ is slim.

Proof. Property (i) follows from Proposition 1.2.1, (ii), together with the fact that the quotient $G_{K_{i}} \rightarrow G_{k_{i}}^{\text {log }}$ may be identified with the quotient of $G_{K_{i}}$ by the (unique) maximal pro- $p$ subgroup of $I_{K_{i}}$. Next, since any morphism of $k_{i}^{\times}$-torsors is necessarily an isomorphism, property (ii) follows by observing that the stated association of coverings to uniformizers is indeed a morphism of $k_{i}^{\times}$-torsors - a tautology, which may by verified by considering the case $N=q_{i}-1$ (where $q_{i}$ is the cardinality of $k_{i}$ ), in which case this tautology amounts to the computation: $\left(\zeta^{1 / N}\right)^{q_{i}}=\zeta \cdot\left(\zeta^{1 / N}\right)$ (for $\left.\zeta \in k_{i}^{\times}\right)$. Finally, property (iii) follows formally from the fact that the quotient $G_{k_{i}}^{\log } / \operatorname{Im}\left(\boldsymbol{\mu}_{\widehat{\mathbb{Z}}}\left(\bar{k}_{i}\right)\right)$ is infinite and acts faithfully on all open subgroups of the closed subgroup $\operatorname{Im}\left(\boldsymbol{\mu}_{\widehat{\mathbb{Z}}}\left(\bar{k}_{i}\right)\right) \subseteq G_{k_{i}}^{\log }$.

In the following, let us denote by $\left(k_{i}^{\log }\right)^{\sim} \rightarrow k_{i}^{\log }$ the "universal covering" of $k_{i}^{\log }$

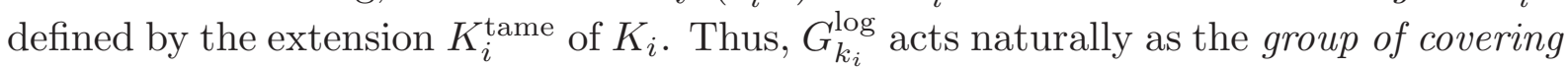
transformations of $\left(k_{i}^{\log }\right)^{\sim} \rightarrow k_{i}^{\log }$. 
Proposition 1.2.4. (The "Grothendieck Conjecture" for the Logarithmic

Point) Suppose that we are given an isomorphism of profinite groups:

$$
\lambda: G_{k_{1}}^{\log } \stackrel{\sim}{\rightarrow} G_{k_{2}}^{\log }
$$

Then:

(i) We have: $\left|k_{1}\right|=\left|k_{2}\right| ; p_{1}=p_{2}$. Thus, (in the remainder of this proposition and its proof) we shall write $p \stackrel{\text { def }}{=} p_{1}=p_{2}$.

(ii) $\lambda$ preserves the subgroups $\operatorname{Im}\left(\boldsymbol{\mu}_{\widehat{\mathbb{Z}}}\left(\bar{k}_{i}\right)\right) \subseteq G_{k_{i}}^{\log }$ as well as the Frobenius elements in the quotients $G_{k_{i}}^{\log } / \operatorname{Im}\left(\boldsymbol{\mu}_{\widehat{\mathbb{Z}}}\left(\bar{k}_{i}\right)\right)$.

(iii) Assume further that the morphism

$$
\bar{k}_{1}^{\times} \stackrel{\sim}{\rightarrow} \bar{k}_{2}^{\times}
$$

induced by $\lambda$ (by thinking of $\bar{k}_{i}^{\times}$as $\boldsymbol{\mu}_{\mathbb{Q} / \mathbb{Z}}\left(\bar{k}_{i}\right)$ ) arises from an isomorphism of fields $\bar{\sigma}: \bar{k}_{1} \stackrel{\sim}{\rightarrow} \bar{k}_{2}$. Then there exists a unique commutative diagram

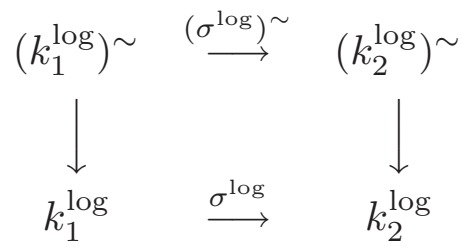

of log schemes, compatible with the natural action of $G_{k_{i}}^{\log }$ on $\left(k_{i}^{\log }\right)^{\sim}($ for $i=1,2)$, in which the vertical morphisms are the natural morphisms, and the horizontal morphisms are isomorphisms for which the morphisms on the underlying schemes are those induced by $\bar{\sigma}$.

Proof. Property (i) follows by observing that $p_{i}$ is the unique prime number such that 1 plus the cardinality of the torsion subgroup of $\left(G_{k_{i}}^{\log }\right)^{\text {ab }}$ - i.e., the cardinality of $k_{i}$ - is equal to a power of $p_{i}$. Property (ii) follows by thinking of the quotients $G_{k_{i}}^{\log } / \operatorname{Im}\left(\boldsymbol{\mu}_{\widehat{\mathbb{Z}}}\left(\bar{k}_{i}\right)\right)$ as the quotients of $G_{k_{i}}^{\text {log }}$ obtained by forming the quotient of $\left(G_{k_{i}}^{\log }\right)^{\text {ab }}$ by its torsion subgroup, and then using that the Frobenius element is the unique element that acts on the abelian group $\operatorname{Im}\left(\boldsymbol{\mu}_{\widehat{\mathbb{Z}}}\left(\bar{k}_{i}\right)\right)$ via multiplication by $\left|k_{1}\right|=\left|k_{2}\right|$. As for (iii), the morphism $\sigma^{\log }$ is the unique logarithmic extension of $\sigma$ whose induced morphism $U_{1} \stackrel{\sim}{\rightarrow} U_{2}$ is the morphism obtained (cf. Proposition 1.2.3, (ii)) by considering the morphism induced by $\lambda$ between the $H^{1}\left(\widehat{\mathbb{Z}}, \boldsymbol{\mu}_{\widehat{\mathbb{Z}}}\left(\bar{k}_{i}\right)\right)=k_{i}^{\times}$-torsors $H^{1}\left(G_{k_{i}}^{\text {log }}, \boldsymbol{\mu}_{\widehat{\mathbb{Z}}}\left(\bar{k}_{i}\right)\right)^{[1]}$ (for $i=1,2)$ - which are preserved by $\lambda$, by (ii). Note that here we also use (cf. (ii)) that the Frobenius element $\in \widehat{\mathbb{Z}}$ is preserved, since this element is necessary to ensure the compatibility of the identifications

$$
H^{1}\left(\widehat{\mathbb{Z}}, \boldsymbol{\mu}_{\widehat{\mathbb{Z}}}\left(\bar{k}_{i}\right)\right)=k_{i}^{\times}
$$


(cf. Proposition 1.2.3, (ii)). The morphism $\left(\sigma^{\log }\right)^{\sim}$ is obtained by applying this construction of " $\sigma$ log" to the various finite log étale coverings of $k_{i}^{\log }$ obtained by considering various open subgroups of $G_{k_{i}}^{\mathrm{log}}$. Here, the transition morphisms among coverings are induced by the Verlagerung, as in the proof of Proposition 1.2.1. Finally, the uniqueness of the lifting $\left(\sigma^{\log }\right)^{\sim}$ of $\sigma^{\log }$ is a formal consequence of the fact that $G_{k_{i}}^{\log }$ is center-free (cf. Proposition 1.2.3, (iii)).

\section{$\S 1.3$. The Anabelian Geometry of Hyperbolic Curves}

\section{Characteristic Zero:}

Let $K$ be a field of characteristic 0 whose absolute Galois group is slim. Let $X$ be a hyperbolic curve of type $(g, r)$ over $S \stackrel{\text { def }}{=} \operatorname{Spec}(K)$. Fix an algebraic closure $\bar{K}$ of $K$ and write $\bar{s}: \operatorname{Spec}(\bar{K}) \rightarrow S ; G_{K} \stackrel{\text { def }}{=} \operatorname{Gal}(\bar{K} / K)$. Let $\bar{x} \in X(\bar{K})$ be a $\bar{K}$-valued point of $X$ lying over $\bar{s}$. Then, setting $\Pi_{X} \stackrel{\text { def }}{=} \pi_{1}(X, \bar{x})$, we obtain an exact sequence

$$
1 \rightarrow \Delta_{X} \rightarrow \Pi_{X} \rightarrow G_{K} \rightarrow 1
$$

which determines a well-defined continous homomorphism

$$
G_{K} \rightarrow \operatorname{Out}\left(\Delta_{X}\right)
$$

to the group of outer automorphisms $\operatorname{Out}\left(\Delta_{X}\right)$ of $\Delta_{X}$.

Lemma 1.3.1. (Slimness of Geometric and Arithmetic Fundamental Groups) The profinite groups $\Delta_{X}, \Pi_{X}$ are slim.

Proof. The slimness of $\Pi_{X}$ is a formal consequence of the slimness of $\Delta_{X}$ and our assumption that $G_{K}$ is slim. Thus, it remains to prove that $\Delta_{X}$ is slim. One approach to proving this fact is given in [Naka1], Corollary 1.3.4. Another approach is the following: Let $H \subseteq \Delta_{X}$ be an open normal subgroup for which the associated covering $X_{H} \rightarrow X_{\bar{K}} \stackrel{\text { def }}{=} X \times_{K} \bar{K}$ is such that $X_{H}$ is a curve of genus $\geq 2$. Thus, $H^{\text {ab }}$ may be thought of as the profinite Tate module associated to the generalized Jacobian of the singular curve obtained from the unique smooth compactification of $X_{H}$ by identifying the various cusps (i.e., points of the compactification not lying in $X_{H}$ ) to a single point. In particular, if conjugation by an element $\delta \in \Delta_{X}$ induces the trivial action on $H^{\mathrm{ab}}$, then we conclude that the image of $\delta$ in $\Delta_{X} / H$ induces the trivial action on the generalized Jacobian just discussed, hence on $X_{H}$ itself. But this implies that $\delta \in H$. By taking $H$ to be sufficiently small, we thus conclude that $\delta=1$. 
In particular, it follows formally from Lemma 1.3.1 that:

Corollary 1.3.2. (A Natural Exact Sequence) We have a natural exact sequence of profinite groups:

$$
1 \rightarrow \Delta_{X} \rightarrow \operatorname{Aut}\left(\Delta_{X}\right) \rightarrow \operatorname{Out}\left(\Delta_{X}\right) \rightarrow 1
$$

In particular, by pulling back this exact sequence, one may recover the exact sequence $1 \rightarrow \Delta_{X} \rightarrow \Pi_{X} \rightarrow G_{K} \rightarrow 1$ entirely group-theoretically from the outer Galois representation $G_{K} \rightarrow \operatorname{Out}\left(\Delta_{X}\right)$.

One example of the sort of " $K$ " under consideration is the case of a "sub-p-adic field":

Corollary 1.3.3. (Slimness of Sub-p-adic Fields) The absolute Galois group of a sub-p-adic field (i.e., a field isomorphic to a subfield of a finitely generated field extension of $\mathbb{Q}_{p}$, for some prime number $p$ ) is slim.

Proof. This fact is implied by the argument of the proof of [Mzk6], Lemma 15.8.

In [Mzk6], the author (essentially) proved the following result (cf. [Mzk6], Theorem A, for a stronger version that treats arbitrary dominant morphisms and open group homomorphisms [i.e., which are not necessarily isomorphisms]):

Theorem 1.3.4. ("Sub- $p$-adic Profinite Grothendieck Conjecture") Suppose that $K$ is a sub-p-adic field, and that $X$ and $Y$ are hyperbolic curves over $K$. Denote by $\operatorname{Isom}_{K}(X, Y)$ the set of $K$-isomorphisms $X \stackrel{\sim}{\rightarrow} Y$; by $\operatorname{Isom}_{G_{K}}^{\text {Out }}\left(\Delta_{X}, \Delta_{Y}\right)$ the set of outer isomorphisms between the two profinite groups in parentheses that are compatible with the respective outer actions of $G_{K}$. Then the natural map

$$
\operatorname{Isom}_{K}(X, Y) \rightarrow \operatorname{Isom}_{G_{K}}^{\mathrm{Out}}\left(\Delta_{X}, \Delta_{Y}\right)
$$

is bijective.

Thus, by combining Theorems 1.1.3; 1.3.4; Lemma 1.1.4, (i), we obtain the following:

Corollary 1.3.5. (Absolute Grothendieck Conjecture over Number Fields) Let $K, L$ be number fields; $X$ (respectively, $Y$ ) a hyperbolic curve over $K$ (respectively, L). Denote by $\operatorname{Isom}(X, Y)$ the set of scheme isomorphisms $X \stackrel{\sim}{\rightarrow} Y$; by 
Isom ${ }^{\text {Out }}\left(\Pi_{X}, \Pi_{Y}\right)$ the set of outer isomorphisms between the two profinite groups in parentheses. Then the natural map

$$
\operatorname{Isom}(X, Y) \rightarrow \operatorname{Isom}^{\text {Out }}\left(\Pi_{X}, \Pi_{Y}\right)
$$

is bijective.

Remark 1.3.5.1. Since the analogue of Theorem 1.1.3 in the $p$-adic local case is false, it seems unlikely to the author at the time of writing that the analogue of Corollary 1.3.5 should hold over $p$-adic local fields.

Remark 1.3.5.2. In fact, the statement of Corollary 1.3 .5 holds when $K$ is taken to be an arbitrary finitely generated field extension of $\mathbb{Q}$. This follows by applying a theorem of F. Pop (in place of the Neukirch-Uchida theorem) — cf. the discussion on [NSW], p. 677, for more details.

One interesting result in the present context is the following, due to M. Matsumoto (cf. [Mtmo], Theorems 2.1, 2.2):

Theorem 1.3.6. (Kernels of Outer Galois Representations) Let $X$ be an affine hyperbolic curve over a sub-complex field $K$ - i.e., a field isomorphic to a subfield of the field of complex numbers. Then the kernel of the resulting outer Galois representation

$$
G_{K} \rightarrow \operatorname{Out}\left(\Delta_{X}\right)
$$

is contained in the kernel of the natural homomorphism $G_{K} \rightarrow G_{\mathbb{Q}}$.

Remark 1.3.6.1. Thus, in particular, when $K$ is a number field, the homomorphism $G_{K} \rightarrow \operatorname{Out}\left(\Delta_{X}\right)$ is injective. This injectivity was first proven by Belyi in the case of hyperbolic curves of type $(g, r)=(0,3)$. It was then conjectured by Voevodskii to be true for all (hyperbolic) $(g, r)$ and proven by Voevodskii to be true for $g=1$. Finally, it was proven by Matsumoto to hold for all $(g, r)$ such that $r>0$. To the knowledge of the author, the proper case remains open at the time of writing. We refer to the discussion surrounding [Mtmo], Theorem 2.1, for more details on this history.

Remark 1.3.6.2. One interesting aspect of the homomorphism appearing in Theorem 1.3.6 is that it allows one to interpret Theorem 1.3.4 (when $X=Y$ ) as a computation of the centralizer of the image of this homomorphism $G_{K} \rightarrow \operatorname{Out}\left(\Delta_{X}\right)$. This point of view is surveyed in detail in [Naka2]. 
Next, we would like to discuss various properties of the inertia groups of the cusps of a hyperbolic curve. For every cusp $x$ of $X_{\bar{K}} \stackrel{\text { def }}{=} X \times_{K} \bar{K}$ - i.e., point of the unique smooth compactification of $X_{\bar{K}}$ over $\bar{K}$ that does not lie in $X_{\bar{K}}$ - we have an associated inertia group (abstractly isomorphic to $\widehat{\mathbb{Z}}$ )

$$
I_{x} \subseteq \Delta_{X}
$$

(well-defined, up to conjugation). If $l$ is any prime number, then let us denote the maximal pro-l quotient of a profinite group by means of a superscript " $(l)$ ". Thus, we also obtain an inertia group $I_{x}^{(l)} \subseteq \Delta_{X}^{(l)}$ (abstractly isomorphic to $\mathbb{Z}_{l}$ ).

Lemma 1.3.7. (Commensurable Terminality of Inertia Groups) The subgroups $I_{x}^{(l)} \subseteq \Delta_{X}^{(l)}, I_{x} \subseteq \Delta_{X}$ are commensurably terminal.

Proof. Indeed, let $\sigma$ be an element of the commensurator. If the asserted commensurable terminality is false, then by projecting to a finite quotient, we may assume that we have a finite Galois covering

$$
Z \rightarrow X_{\bar{K}}
$$

(of degree a power of $l$ in the pro- $l$ case), together with a cusp $z$ of $Z$ such that: (i) $z$ maps to $x$; (ii) $z \neq z^{\sigma}$; (iii) $z, z^{\sigma}$ have conjugate inertia groups in $\Delta_{Z}$. We may also assume (by taking $\Delta_{Z} \subseteq \Delta_{X}$ to be sufficiently small) that $Z$ has genus $\geq 2$ and admits a cusp $z^{\prime} \neq z, z^{\sigma}$. Then it is easy to see that $Z$ admits an infinite abelian (pro- $l$, in the pro- $l$ case) covering which is totally ramified at $z, z^{\prime}$, but not at $z^{\sigma}$. But this contradicts property (iii).

Remark 1.3.7.1. As was pointed out to the author by the referee, in the case $r \geq 2$, the assertion of Lemma 1.3.7 is a formal consequence of Theorem $\mathrm{B}^{\prime}$ of $[\mathrm{HR}]$.

Now, let us assume that we are given two hyperbolic curves $\left(X_{i}\right)_{K_{i}}$ (for $i=1,2$ ), each defined over a finite extension $K_{i}$ of $\mathbb{Q}_{p_{i}}$. Let us write $q_{i}$ for the cardinality of the residue field of $K_{i}$. Choose basepoints for the $\left(X_{i}\right)_{K_{i}}$ and denote the resulting fundamental groups by $\Pi_{\left(X_{i}\right)_{K_{i}}}$. Also, let us denote the unique proper smooth curve over $K_{i}$ that compactifies $\left(X_{i}\right)_{K_{i}}$ by $\left(Y_{i}\right)_{K_{i}}$. Suppose, moreover, that we are given an isomorphism

$$
\alpha_{X}: \Pi_{\left(X_{1}\right)_{K_{1}}} \stackrel{\sim}{\rightarrow} \Pi_{\left(X_{2}\right)_{K_{2}}}
$$

of profinite groups.

Lemma 1.3.8. (Group-Theoreticity of Arithmetic Quotients) The isomorphism $\alpha_{X}$ is necessarily compatible with the quotients $\Pi_{\left(X_{i}\right)_{K_{i}}} \rightarrow G_{K_{i}}$. 
Proof. This follows formally from Lemmas 1.1.4, 1.1.5.

Thus, Lemma 1.3.8, Proposition 1.2.1, (v), imply that $q_{1}=q_{2}$.

Lemma 1.3.9. (Group-Theoreticity of the Cusps) The types $\left(g_{i}, r_{i}\right)$ of the hyperbolic curves $\left(X_{i}\right)_{K_{i}}$ coincide. In particular, for any prime number $l, \alpha_{X}$ maps inertia groups of cusps in $\Delta_{X_{1}}$ (respectively, $\Delta_{X_{1}}^{(l)}$ ) to inertia groups of cusps in $\Delta_{X_{2}}$ (respectively, $\left.\Delta_{X_{2}}^{(l)}\right)$.

Proof. Whether or not $r_{i}=0$ may be determined by considering whether or not $\Delta_{X_{i}}$ is free as a profinite group. When $r_{i}>0$, one may compute $r_{i}$ by considering the weight - i.e., the number $w$ such that the eigenvalues of the action are algebraic numbers of archimedean absolute value $q_{i}^{w}$ - of the action of the Frobenius element $\in G_{k_{i}}$ (cf. Proposition 1.2.1, (iv)) as follows: First, we observe that (as is well-known) the weights of the action of Frobenius on $\Delta_{X_{i}}^{\mathrm{ab}} \otimes \mathbb{Q}_{l}$ (where $l$ is a prime number distinct from $p_{1}$, $p_{2}$ ) belong to the set $\left\{0,1, \frac{1}{2}\right\}$. (Here, we compute weights by choosing a lifting of the Frobenius element $\in G_{k_{i}}$ to $G_{K_{i}}$. Since (as is well-known) the action of the inertia subgroup of $G_{K_{i}}$ on $\Delta_{X_{i}}^{\mathrm{ab}} \otimes \mathbb{Q}_{l}$ is quasi-unipotent (i.e., unipotent on an open subgroup of this inertia subgroup), it follows immediately that the weights are independent of the choice of lifting.) Now if $M$ is a $\mathbb{Q}_{l}$-vector space on which Frobenius acts, let us write

$$
M^{\mathrm{wt} w}
$$

for the $\mathbb{Q}_{l}$-subspace of $M$ on which Frobenius acts with eigenvalues of weight $w$. Then, setting $I_{i} \stackrel{\text { def }}{=} \operatorname{Ker}\left(\Delta_{X_{i}}^{\mathrm{ab}} \otimes \mathbb{Q}_{l} \rightarrow \Delta_{Y_{i}}^{\mathrm{ab}} \otimes \mathbb{Q}_{l}\right)$, we have:

$$
\begin{aligned}
r_{i}-1 & =\operatorname{dim}_{\mathbb{Q}_{l}}\left(I_{i}\right)=\operatorname{dim}_{\mathbb{Q}_{l}}\left(I_{i}^{\mathrm{wt} 1}\right) \\
& =\operatorname{dim}_{\mathbb{Q}_{l}}\left(\Delta_{X_{i}}^{\mathrm{ab}} \otimes \mathbb{Q}_{l}\right)^{\mathrm{wt} 1}-\operatorname{dim}_{\mathbb{Q}_{l}}\left(\Delta_{Y_{i}}^{\mathrm{ab}} \otimes \mathbb{Q}_{l}\right)^{\mathrm{wt} 1} \\
& =\operatorname{dim}_{\mathbb{Q}_{l}}\left(\Delta_{X_{i}}^{\mathrm{ab}} \otimes \mathbb{Q}_{l}\right)^{\mathrm{wt} 1}-\operatorname{dim}_{\mathbb{Q}_{l}}\left(\Delta_{Y_{i}}^{\mathrm{ab}} \otimes \mathbb{Q}_{l}\right)^{\mathrm{wt} 0} \\
& =\operatorname{dim}_{\mathbb{Q}_{l}}\left(\Delta_{X_{i}}^{\mathrm{ab}} \otimes \mathbb{Q}_{l}\right)^{\mathrm{wt} 1}-\operatorname{dim}_{\mathbb{Q}_{l}}\left(\Delta_{X_{i}}^{\mathrm{ab}} \otimes \mathbb{Q}_{l}\right)^{\mathrm{wt} 0}
\end{aligned}
$$

(where the fourth equality follows from the auto-duality (up to a Tate twist) of $\Delta_{Y_{i}}^{\mathrm{ab}} \otimes$ $\mathbb{Q}_{l}$; and the second and fifth equalities follow from the fact that Frobenius acts on $I_{i}$ with weight 1). On the other hand, the quantities appearing in the final line of this sequence of equalities are all "group-theoretic". Thus, we conclude that $r_{1}=r_{2}$. Since $\operatorname{dim}_{\mathbb{Q}_{l}}\left(\Delta_{X_{i}}^{\mathrm{ab}} \otimes \mathbb{Q}_{l}\right)=2 g_{i}-1+r_{i}$ (respectively, $=2 g_{i}$ ) when $r_{i}>0$ (respectively, when $\left.r_{i}=0\right)$, this implies that $g_{1}=g_{2}$, as desired.

Finally, the statement concerning preservation of inertia groups follows formally from the fact that " $r_{i}$ is group-theoretic" (by applying this fact to coverings of $X_{i}$ ). 
Indeed, let $l$ be a prime number (possibly equal to $p_{1}$ or $p_{2}$ ). Since $r_{i}$ may be recovered group-theoretically, given any finite étale coverings

$$
Z_{i} \rightarrow V_{i} \rightarrow X_{i}
$$

such that $Z_{i}$ is Galois, of degree a power of $l$, over $V_{i}$, one may determine grouptheoretically whether or not $Z_{i} \rightarrow V_{i}$ is "totally ramified at a single point of $Z_{i}$ and unramified elsewhere", since this condition is easily verified to be equivalent to the equality:

$$
r_{Z_{i}}=\operatorname{deg}\left(Z_{i} / V_{i}\right) \cdot\left(r_{V_{i}}-1\right)+1
$$

Moreover, the group-theoreticity of this condition extends immediately to the case of pro-l coverings $Z_{i} \rightarrow V_{i}$. Thus, by Lemma 1.3.7, we conclude that the inertia groups of cusps in $\left(\Delta_{X_{i}}\right)^{(l)}$ (i.e., the maximal pro-l quotient of $\Delta_{X_{i}}$ ) may be characterized (grouptheoretically!) as the maximal subgroups of $\left(\Delta_{X_{i}}\right)^{(l)}$ that correspond to (profinite) coverings satisfying this condition.

In particular, (by Lemma 1.3.7) the set of cusps of $X_{i}$ may be reconstructed (grouptheoretically!) as the set of $\left(\Delta_{X_{i}}\right)^{(l)}$-orbits (relative to the action via conjugation) of such inertia groups in $\left(\Delta_{X_{i}}\right)^{(l)}$. Thus, by applying this observation to arbitrary finite étale coverings of $X_{i}$, we recover the inertia subgroups of the cusps of $\Delta_{X_{i}}$ as the subgroups that fix some cusp of the universal covering $\widetilde{X}_{i} \rightarrow X_{i}$ of $X_{i}$ determined by the basepoint in question. This completes the proof.

Remark 1.3.9.1. As was pointed out to the author by the referee, the argument given in the second paragraph of the proof of Lemma 1.3.9 may be replaced by the more group-theoretic argument of [Tama2], Proposition 2.4.

\section{Positive Characteristic:}

For $i=1,2$, let $k_{i}$ be a finite field of characteristic $p ; X_{i}$ a hyperbolic curve over $k_{i}$. Choose a universal tamely ramified (i.e., at the punctures of $X_{i}$ ) covering $\widetilde{X}_{i} \rightarrow X_{i}$ of $X_{i}$; write

$$
\Pi_{X_{i}}^{\text {tame }} \stackrel{\text { def }}{=} \operatorname{Gal}\left(\widetilde{X}_{i} / X_{i}\right)
$$

for the corresponding fundamental groups. Thus, we obtain exact sequences:

$$
1 \rightarrow \Delta_{X_{i}}^{\text {tame }} \rightarrow \Pi_{X_{i}}^{\text {tame }} \rightarrow G_{k_{i}} \rightarrow 1
$$

(where $G_{k_{i}}$ is the absolute Galois group of $k_{i}$ determined by $\widetilde{X}_{i}$ ). As is well-known, the Frobenius element determines a natural isomorphism $\widehat{\mathbb{Z}} \cong G_{k_{i}}$.

Lemma 1.3.10. (Slimness of Fundamental Groups) For $i=1,2$, the profinite groups $\Delta_{X_{i}}^{\text {tame }}, \Pi_{X_{i}}^{\text {tame }}$ are slim. 


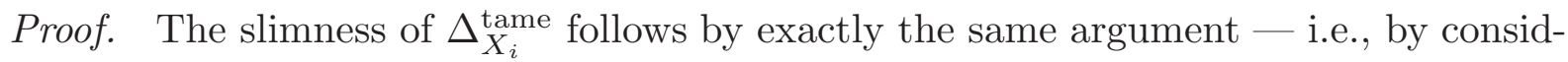
ering the action of $\Delta_{X_{i}}^{\text {tame }}$ on abelianizations of open subgroups - as that given in the proof of Lemma 1.3.1. [Alternatively, as was pointed out to the author by the referee,

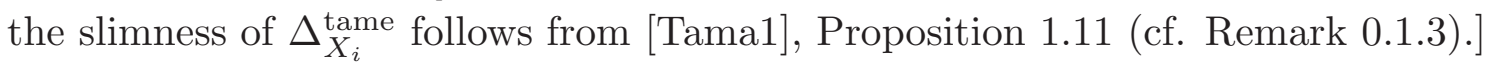

By a similar argument, the slimness of $\Pi_{X_{i}}^{\text {tame }}$ follows formally from:

(i) the slimness of $\Delta_{X_{i}}^{\text {tame; }}$

(ii) some positive power of the character of $G_{k_{i}}$ arising from the determinant of the prime-to-p portion of $\left(\Delta_{X_{i}}^{\text {tame }}\right)^{\text {ab }}$ coincides with some positive power of the cyclotomic character.

(Here, we note (ii) is sufficient to deal with both the "l-primary portion" of $\widehat{\mathbb{Z}} \cong G_{k_{i}}$ (for $l \neq p$ ) and the "p-primary portion" of $\widehat{\mathbb{Z}} \cong G_{k_{i}}$.)

Remark 1.3.10.1. Property (ii) in the proof of Lemma 1.3 .10 was substantially simplified by a suggestion made to the author by the referee.

The following fundamental result is due to A. Tamagawa (cf. [Tama1], Theorem 4.3):

Theorem 1.3.11. (The Grothendieck Conjecture for Affine Hyperbolic Curves over Finite Fields) Assume, for $i=1,2$, that $X_{i}$ is affine. Then the natural map

$$
\operatorname{Isom}\left(\widetilde{X}_{1} / X_{1}, \widetilde{X}_{2} / X_{2}\right) \rightarrow \operatorname{Isom}\left(\Pi_{X_{1}}^{\text {tame }}, \Pi_{X_{2}}^{\text {tame }}\right)
$$

(from scheme-theoretic isomorphisms $\widetilde{X}_{1} \stackrel{\sim}{\rightarrow} \widetilde{X}_{2}$ lying over an isomorphism $X_{1} \stackrel{\sim}{\rightarrow} X_{2}$ to isomorphisms of profinite groups $\Pi_{X_{1}}^{\text {tame }} \stackrel{\sim}{\rightarrow} \Pi_{X_{2}}^{\text {tame }}$ ) is bijective.

Finally, we observe that, just as in the characteristic zero case, inertia groups of cusps are commensurably terminal: If $x_{i}$ is a cusp of $\left(X_{i}\right)_{\bar{k}_{i}} \stackrel{\text { def }}{=} X_{i} \times_{k_{i}} \bar{k}_{i}$, then we have an associated inertia group (abstractly isomorphic to $\widehat{\mathbb{Z}}^{\prime}$ )

$$
I_{x_{i}} \subseteq \Delta_{X_{i}}^{\mathrm{tame}}
$$

(well-defined, up to conjugation). If $l$ is any prime number distinct from $p$, then we also obtain an inertia group $I_{x_{i}}^{(l)} \subseteq\left(\Delta_{X_{i}}^{\text {tame }}\right)^{(l)}$ (abstractly isomorphic to $\mathbb{Z}_{l}$ ).

Lemma 1.3.12. (Commensurable Terminality of Inertia Groups) The subgroups $I_{x_{i}}^{(l)} \subseteq\left(\Delta_{X_{i}}^{\text {tame }}\right)^{(l)}, I_{x_{i}} \subseteq \Delta_{X_{i}}^{\text {tame }}$ are commensurably terminal. 
Proof. The proof is entirely similar to that of Lemma 1.3.7. [Alternatively, as was pointed out to the author by the referee, the assertion concerning $I_{x_{i}} \subseteq \Delta_{X_{i}}^{\text {tame }}$ follows from [Tama2], Lemma 2.1, while the assertion concerning $I_{x_{i}}^{(l)} \subseteq\left(\Delta_{X_{i}}^{\text {tame }}\right)^{(l)}$ follows, at least in the case $r \geq 2$, formally from Theorem $\mathrm{B}^{\prime}$ of [HR].] $\bigcirc$

\section{Section 2: Reconstruction of the Logarithmic Special Fiber}

For $i=1,2$, let $K_{i}$ be a finite extension of $\mathbb{Q}_{p_{i}}$ (cf. $\left.\S 1.2\right)$, and suppose that we are

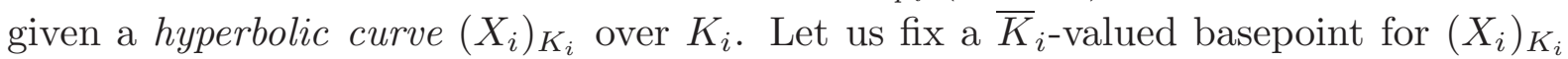
and denote the resulting fundamental group $\pi_{1}\left(\left(X_{i}\right)_{K_{i}}\right)$ by $\Pi_{\left(X_{i}\right)_{K_{i}}}$. Suppose, moreover,

that we are given an isomorphism $\alpha_{X}: \Pi_{\left(X_{1}\right)_{K_{1}}} \stackrel{\sim}{\rightarrow} \Pi_{\left(X_{2}\right)_{K_{2}}}$, which, by Lemma 1.3.8, necessarily fits into a commutative diagram

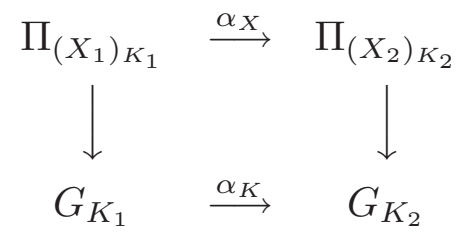

where the vertical morphisms are the natural ones, and the horizontal morphisms are assumed to be isomorphisms. Note that by Proposition 1.2.1, (i), this already implies that $p_{1}=p_{2}$; set $p \stackrel{\text { def }}{=} p_{1}=p_{2}$. That such a diagram necessarily arises "geometrically" follows from the main theorem of [Mzk6] (cf. Theorem 1.3.4) - if one assumes that $\alpha_{K}$ arises geometrically (i.e., from an isomorphism of fields $K_{1} \stackrel{\sim}{\rightarrow} K_{2}$ ). In this $\S$, we would like to investigate what one can say in general (i.e., without assuming that $\alpha_{K}$ arises geometrically) concerning this sort of commutative diagram. In some sense, all the key arguments that we use here are already present in [Mzk4], except that there, these arguments were applied to prove different theorems. Thus, in the following discussion, we explain how the same arguments may be used to prove Theorem 2.7 below.

Let us denote the type of the hyperbolic curve $\left(X_{i}\right)_{K_{i}}$ by $\left(g_{i}, r_{i}\right)$. Also, we shall denote the geometric fundamental group by

$$
\Delta_{X_{i}} \stackrel{\text { def }}{=} \operatorname{Ker}\left(\Pi_{\left(X_{i}\right)_{K_{i}}} \rightarrow G_{K_{i}}\right)
$$

and the unique proper smooth curve over $K_{i}$ that compactifies $\left(X_{i}\right)_{K_{i}}$ by $\left(Y_{i}\right)_{K_{i}}$.

Lemma 2.1. (Group-Theoreticity of Stability) $\left(X_{1}\right)_{K_{1}}$ has stable reduction if and only if $\left(X_{2}\right)_{K_{2}}$ does.

Proof. This follows (essentially) from the well-known stable reduction criterion: That is to say, $\left(X_{i}\right)_{K_{i}}$ has stable reduction if and only if the inertia subgroup of $G_{K_{i}}$ acts 
unipotently on $\Delta_{Y_{i}}^{\mathrm{ab}} \otimes \widehat{\mathbb{Z}}^{\prime}$ and trivially on the (finite) set of conjugacy classes of inertia groups of cusps in $\Delta_{X_{i}}$ (i.e., the set of cusps of $\left(X_{i}\right)_{K_{i}} \otimes_{K_{i}} \bar{K}_{i}-$ cf. Lemma 1.3.9). [Note that this condition is group-theoretic, by Proposition 1.2.1, (ii).]

Now let us assume that $\left(X_{i}\right)_{K_{i}}$ has stable reduction over $\mathcal{O}_{K_{i}}$. Denote the stable model of $\left(X_{i}\right)_{K_{i}}$ over $\mathcal{O}_{K_{i}}$ by:

$$
\left(\mathcal{X}_{i}\right)_{\mathcal{O}_{K_{i}}} \rightarrow \operatorname{Spec}\left(\mathcal{O}_{K_{i}}\right)
$$

Here, in the case where $r_{i}>0$, we mean by the term "stable model" the complement of the marked points in the unique stable pointed curve over $\mathcal{O}_{K_{i}}$ that extends the pointed curve over $K_{i}$ determined by $\left(X_{i}\right)_{K_{i}}$. Then, by the theory of [Mzk4], $\S 2,8$, there exists a well-defined quotient

$$
\Pi_{\left(X_{i}\right)_{K_{i}}} \rightarrow \Pi_{\left(X_{i}\right)_{K_{i}}}^{\mathrm{adm}}
$$

whose finite quotients correspond to (subcoverings of) admissible coverings of the result of base-changing $\left(\mathcal{X}_{i}\right)_{\mathcal{O}_{K_{i}}}$ to rings of integers of tamely ramified extensions of $K_{i}$. In particular, we have a natural exact sequence:

$$
1 \rightarrow \Delta_{X_{i}}^{\mathrm{adm}} \rightarrow \Pi_{\left(X_{i}\right)_{K_{i}}}^{\mathrm{adm}} \rightarrow G_{k_{i}}^{\mathrm{log}} \rightarrow 1
$$

(where $\Delta_{X_{i}}^{\mathrm{adm}}$ is defined so as to make the sequence exact). Moreover, $\Pi_{\left(X_{i}\right)_{K_{i}}}^{\mathrm{adm}}$ itself admits a natural quotient (cf. [Mzk4], §3)

$$
\Pi_{\left(X_{i}\right)_{K_{i}}} \rightarrow \Pi_{\left(X_{i}\right)_{K_{i}}}^{\mathrm{adm}} \rightarrow \Pi_{\left(X_{i}\right)_{K_{i}}}^{\mathrm{et}}
$$

whose finite quotients correspond to coverings of $\left(X_{i}\right)_{K_{i}}$ that extend to finite étale coverings of $\left(\mathcal{X}_{i}\right)_{\mathcal{O}_{K_{i}}}$ which are tamely ramified at the cusps. In particular, we have a natural exact sequence:

$$
1 \rightarrow \Delta_{X_{i}}^{\mathrm{et}} \rightarrow \Pi_{\left(X_{i}\right)_{K_{i}}}^{\mathrm{et}} \rightarrow G_{k_{i}} \rightarrow 1
$$

(where $\Delta_{X_{i}}^{\text {et }}$ is defined so as to make the sequence exact).

\section{Lemma 2.2. (Admissible and Étale Quotients)}

(i) The profinite groups $\Pi_{\left(X_{i}\right)_{K_{i}}}, \Pi_{\left(X_{i}\right)_{K_{i}}}^{\mathrm{adm}}$, and $\Delta_{X_{i}}^{\mathrm{adm}}$ are all slim.

(ii) The morphism $\alpha_{X}$ is compatible with the quotients

$$
\Pi_{\left(X_{i}\right)_{K_{i}}} \rightarrow \Pi_{\left(X_{i}\right)_{K_{i}}}^{\mathrm{adm}} \rightarrow \Pi_{\left(X_{i}\right)_{K_{i}}}^{\mathrm{et}}
$$

of $\Pi_{\left(X_{i}\right)_{K_{i}}}$. 
Proof. We begin with assertion (i). The slimness of $\Pi_{\left(X_{i}\right)_{K_{i}}}$ follows formally from Theorem 1.1.1, (ii); Lemma 1.3.1. Next, let us assume that $\Delta_{X_{i}}^{\operatorname{adm}}$ has already been shown to be slim. Then it follows formally from Proposition 1.2.3, (iii), that $\Pi_{\left(X_{i}\right)_{K_{i}}}^{\mathrm{adm}}$ is slim.

Thus, to complete the proof of assertion (i), it suffices to show that $\Delta_{X_{i}}^{\text {adm }}$ is slim. We argue as in Lemma 1.3.1, i.e., we consider the covering associated to an open normal subgroup $H \subseteq \Delta_{X_{i}}^{\mathrm{adm}}$. To simplify notation, we assume that $\left(\mathcal{X}_{i}\right)_{k_{i}}$ is "that covering"; then it suffices to show (cf. the proof of Lemma 1.3.1) that any automorphism $\left(k_{i}\right.$-linear) $\sigma$ of $\left(\mathcal{X}_{i}\right)_{k_{i}}$ which acts trivially on $\left(\Delta_{X_{i}}^{\mathrm{adm}}\right)^{\mathrm{ab}}$ is itself trivial. We may assume without loss of generality [i.e., by taking $H$ to be sufficiently small] that $\left(\mathcal{X}_{i}\right)_{k_{i}}$ is sturdy (cf. [Mzk4], Definition 1.1) - i.e., that the normalizations of all the geometric irreducible components of $\left(\mathcal{X}_{i}\right)_{k_{i}}$ have genus $\geq 2$. Then by [Mzk4], Proposition 1.3, it follows that $\sigma$ acts trivially on the set of geometric irreducible components of $\left(\mathcal{X}_{i}\right)_{k_{i}}$. In particular, $\sigma$ acts on each such geometric irreducible component individually. Thus, by considering the action of $\sigma$ on the torsion points of the Jacobians of each these geometric irreducible components of $\left(\mathcal{X}_{i}\right)_{k_{i}}$ (cf. the proof of Lemma 1.3.1), we conclude that $\sigma$ acts as the identity on each of these geometric irreducible components, as desired.

Next, we turn to assertion (ii). For $\Pi_{\left(X_{i}\right)_{K_{i}}}^{\mathrm{adm}}$, this follows (essentially) from Proposition 8.4 of [Mzk4] (together with Lemma 1.3.9, which shows that $\alpha_{X}$ preserves the pro- $p$ inertia groups associated to the cusps in $\left.\Pi_{\left(X_{i}\right)_{K_{i}}}\right)$. Of course, in [Mzk4], $K_{1}=K_{2}$ and $\alpha_{K}$ is the identity, but in fact, the only property of $\alpha_{K}$ necessary for the proof of [Mzk4], Proposition 8.4 - which is, in essence, a formal consequence of [Mzk4], Lemma 8.1 (concerning unramified quotients of the $p$-adic Tate module of a semi-abelian variety over a $p$-adic local field) — is that $\alpha_{K}$ preserve the inertia and wild inertia groups (which we know, by Proposition 1.2.1, (ii); Proposition 1.2.3, (i), of the present paper).

Similarly, the portion of assertion (ii) concerning $\Pi_{\left(X_{i}\right)_{K_{i}}}^{\mathrm{et}}$ follows (essentially) from [Mzk4], Proposition 3.2, at least in the case $r_{i}=0$. That is to say, even though $\alpha_{K}$ is not necessarily the identity in the present discussion, the only properties of $\alpha_{K}$ that are necessary for the proof of [Mzk4], Proposition 3.2, are Proposition 1.2.3, (i); Proposition 1.2.4, (ii) (of the present paper).

Finally, to treat the case of arbitrary $r_{i}>0$, we argue as follows: Consider an open normal subgroup $H \subseteq \Pi_{\left(X_{i}\right)_{K_{i}}}^{\mathrm{adm}}$. Then observe that there exists an open normal subgroup $H^{\prime} \subseteq \Pi_{\left(X_{i}\right)_{K_{i}}}^{\mathrm{adm}}$ such that the orders of the finite groups $\Pi_{\left(X_{i}\right)_{K_{i}}}^{\mathrm{adm}} / H, \Pi_{\left(X_{i}\right)_{K_{i}}}^{\mathrm{adm}} / H^{\prime}$ are relatively prime and such that the covering of $\left(\mathcal{X}_{i}\right)_{k_{i}}$ determined by $H^{\prime}$ is sturdy. Moreover, [by the assumption of relative primeness] it follows easily that the covering determined by $H$ is "of étale type" (i.e., arises from a quotient of $\Pi_{\left(X_{i}\right)_{K_{i}}}^{\text {et }}$ ) if and only if it becomes a covering "of étale type" after base-change via the covering determined by $H^{\prime}$. Thus, we conclude that we may assume without loss of generality that $\left(\mathcal{X}_{i}\right)_{k_{i}}$ is sturdy. 
Now, let us observe that since $\left(\mathcal{X}_{i}\right)_{k_{i}}$ is sturdy, it admits admissible coverings "of étale type" with arbitrarily large prime-to-p ramification at the cusps. Thus, [since the condition that an admissible covering be "of étale type" amounts to the condition that there be no ramification at the nodes or at the prime of $\left.\mathcal{O}_{K_{i}}\right]$ we conclude that the covering of $\left(\mathcal{X}_{i}\right)_{k_{i}}$ determined by $H$ is of étale type if and only if, for every open normal $H^{\prime \prime} \subseteq \Pi_{\left(X_{i}\right)_{K_{i}}}^{\mathrm{adm}}$ which has ramification index divisible by the prime-to-p portion of the order of the finite group $\Pi_{\left(X_{i}\right)_{K_{i}}}^{\mathrm{adm}} / H$ at all of the cusps, it becomes a covering of étale type after base-change by the covering determined by $H^{\prime \prime}$. [Indeed, the necessity of this condition is clear; the sufficiency of this condition follows from the existence of coverings of étale type with arbitrarily large prime-to- $p$ ramification at the cusps. Also, we note that by Lemma 1.3.9, this condition is preserved by $\alpha_{X}$.] On the other hand, such base-changed coverings are [by construction] necessarily unramified at the cusps. Thus, the issue of whether or not this base-changed covering is of étale type reduces - by Lemma 1.3.9, which shows that $\alpha_{X}$ preserves the quotient $\Pi_{\left(X_{i}\right)_{K_{i}}} \rightarrow \Pi_{\left(Y_{i}\right)_{K_{i}}}$ — to the " $r_{i}=0$ " case, which has already been resolved. This completes the proof of assertion (ii) in the case $r_{i}>0$.

\section{Lemma 2.3. (Group-Theoreticity of Dual Semi-Graphs of the Special} Fiber) The morphism $\alpha_{X}$ induces an isomorphism

$$
\alpha_{X, \Gamma^{\mathrm{c}}}: \Gamma_{\left(\mathcal{X}_{1}\right)_{k_{1}}}^{\mathrm{c}} \stackrel{\sim}{\rightarrow} \Gamma_{\left(\mathcal{X}_{2}\right)_{k_{2}}}^{\mathrm{c}}
$$

between the "dual semi-graphs with compact structure" (i.e., the usual dual graphs

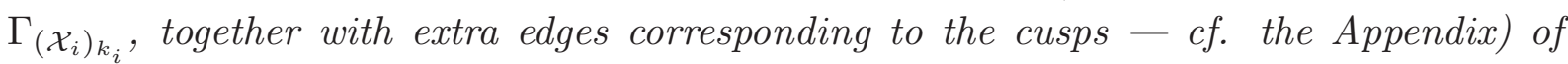
the special fibers $\left(\mathcal{X}_{i}\right)_{k_{i}}$ of $\left(\mathcal{X}_{i}\right)_{\mathcal{O}_{K_{i}}}$. Moreover, $\alpha_{X, \Gamma^{c}}$ is functorial with respect to passage to finite étale coverings of the $\left(X_{i}\right)_{K_{i}}$.

Proof. Indeed, if one forgets about the "compact structure", then this is a formal consequence of Lemma 1.3.9 [which shows that $\alpha_{X}$ preserves the quotient $\Pi_{\left(X_{i}\right)_{K_{i}}} \rightarrow$ $\Pi_{\left(Y_{i}\right)_{K_{i}}}$; Lemma 2.2, (ii), and the theory [concerning the $r_{i}=0$ case] of [Mzk4], $\S 1-5$, summarized in [Mzk4], Corollary 5.3. Even though $\alpha_{K}$ is not necessarily the identity in the present discussion, the only properties of $\alpha_{K}$ that are necessary for the proof of [Mzk4], Corollary 5.3 are Proposition 1.2.3, (i); Proposition 1.2.4, (ii) (of the present paper). That is to say, the point is that the Frobenius element is preserved, which means that the weight filtrations on $l$-adic cohomology (where $l$ is a prime distinct from $p$ ) are, as well.

The compatibility with the "compact structure" follows from the pro- $l$ (where $l \neq p$ ) portion of Lemma 1.3.9, together with the easily verified fact (cf. the proof of Lemma 1.3.7) that the inertia group of a cusp in $\Pi_{\left(X_{i}\right)_{K_{i}}}^{\mathrm{adm}}$ is contained (up to conjugacy) in the decomposition group of a unique irreducible component of $\left(\mathcal{X}_{i}\right)_{k_{i}}$. 
Next, we would like to show that $\alpha_{X}$ is necessarily "of degree 1 ". This is essentially the argument of [Mzk4], Lemma 9.1, but we present this argument in detail below since we are working here under the assumption that $\alpha_{K}$ is arbitrary. For simplicity, we assume until further notice is given that:

$\left(*^{\mathrm{CSSN}}\right) \quad r_{i}=0$ [i.e., the curves are compact] and, moreover, the special fiber $\left(\mathcal{X}_{i}\right)_{k_{i}}$ of $\left(\mathcal{X}_{i}\right)_{\mathcal{O}_{K_{i}}}$ is singular and sturdy (cf. [Mzk4], Definition 1.1) - i.e., the normalizations of all the geometric irreducible components of $\left(\mathcal{X}_{i}\right)_{k_{i}}$ have genus $\geq 2$ - and has a noncontractible dual semi-graph $\Gamma_{\left(\mathcal{X}_{i}\right)_{k_{i}}}^{\mathrm{c}}$ - i.e., this semi-graph is not a tree.

(These conditions may always be achieved by replacing $\left(X_{i}\right)_{K_{i}}$ by a finite étale covering of $\left(X_{i}\right)_{K_{i}}$ - cf. [Mzk4], Lemma 2.9; [Mzk4], the first two paragraphs of the proof of Theorem 9.2.)

We begin by introducing some notation. Write:

$$
\begin{gathered}
V_{i} \stackrel{\text { def }}{=} \Delta_{X_{i}}^{\mathrm{ab}} ; \\
H_{i} \stackrel{\text { def }}{=} H_{1}^{\operatorname{sing}}\left(\Gamma_{\left(\mathcal{X}_{i}\right)_{k_{i}}}^{\mathrm{c}}, \mathbb{Z}\right)=H_{1}^{\text {sing }}\left(\Gamma_{\left(\mathcal{X}_{i}\right)_{k_{i}}}, \mathbb{Z}\right)
\end{gathered}
$$

(where " $H_{1}^{\text {sing" }}$ denotes the first singular homology group). Thus, by considering the coverings of $\left(\mathcal{X}_{i}\right)_{\mathcal{O}_{K_{i}}}$ induced by unramified coverings of the graph $\Gamma_{\left(\mathcal{X}_{i}\right)_{k_{i}}}$, we obtain natural (group-theoretic!) "combinatorial quotients":

$$
V_{i} \rightarrow\left(H_{i}\right)_{\widehat{\mathbb{Z}}} \stackrel{\text { def }}{=} H_{i} \otimes \widehat{\mathbb{Z}}
$$

Lemma 2.4. (Ordinary New Parts, after Raynaud) For a "sufficiently large prime number l" (where "sufficiently large" depends only on $p, g_{i}$ ), and after possibly replacing $K_{i}$ by a finite unramified extension of $K_{i}$, there exists a cyclic étale covering $\left(\mathcal{Z}_{i}\right)_{\mathcal{O}_{K_{i}}} \rightarrow\left(\mathcal{X}_{i}\right)_{\mathcal{O}_{K_{i}}}$ of $\left(\mathcal{X}_{i}\right)_{\mathcal{O}_{K_{i}}}$ of degree $l$ such that the "new part" $V_{i}^{\text {new }} \stackrel{\text { def }}{=}$ $\Delta_{\left(Z_{i}\right)_{K_{i}}}^{\mathrm{ab}} / \Delta_{\left(X_{i}\right)_{K_{i}}}^{\mathrm{ab}}$ of the abelianized geometric fundamental group of $\left(Z_{i}\right)_{K_{i}}$ satisfies the following:

(i) We have an exact sequence:

$$
0 \rightarrow V_{i}^{\text {mlt }} \rightarrow\left(V_{i}^{\text {new }}\right)_{\mathbb{Z}_{p}} \stackrel{\text { def }}{=} V_{i}^{\text {new }} \otimes_{\widehat{\mathbb{Z}}} \mathbb{Z}_{p} \rightarrow V_{i}^{\text {etl }} \rightarrow 0
$$

- where $V_{i}^{\text {etl }}$ is an unramified $G_{K_{i}}$-module, and $V_{i}^{\text {mlt }}$ is the Cartier dual of an unramified $G_{K_{i}}$-module.

(ii) The "combinatorial quotient" of $\Delta_{\left(Z_{i}\right)_{K_{i}}}^{\mathrm{ab}}$ (arising from the coverings of $\left(\mathcal{Z}_{i}\right)_{\mathcal{O}_{K_{i}}}$ induced by unramified coverings of the dual semi-graph of the special fiber of $\left.\left(\mathcal{Z}_{i}\right)_{\mathcal{O}_{K_{i}}}\right)$ induces a nonzero quotient $V_{i}^{\text {new }} \rightarrow\left(H_{i}^{\text {new }}\right)_{\widehat{\mathbb{Z}}}$ of $V_{i}^{\text {new }}$. 
Here, the injection $\Delta_{\left(X_{i}\right)_{K_{i}}}^{\mathrm{ab}} \hookrightarrow \Delta_{\left(Z_{i}\right)_{K_{i}}}^{\mathrm{ab}}$ is the injection induced by pull-back via $\left(Z_{i}\right)_{K_{i}} \rightarrow$ $\left(X_{i}\right)_{K_{i}}$ and Poincaré duality (or, alternatively, by the "Verlagerung").

Proof. Note that since both conditions (i), (ii) are group-theoretic, they may be realized simultaneously for $i=1,2$. Now to satisfy condition (i), it suffices - cf., e.g., the

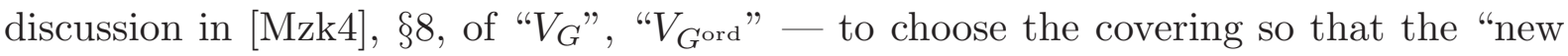
parts" of the Jacobians of the irreducible components of the special fiber of $\left(\mathcal{Z}_{i}\right)_{\mathcal{O}_{K_{i}}}$ are all ordinary. That this is possible for $l$ sufficiently large is a consequence of a theorem of Raynaud (as formulated, for instance, in [Tama1], Lemma 1.9). Then let us observe that, so long as we choose the étale covering $\left(\mathcal{Z}_{i}\right)_{\mathcal{O}_{K_{i}}} \rightarrow\left(\mathcal{X}_{i}\right)_{\mathcal{O}_{K_{i}}}$ so that it is nontrivial over every irreducible component of $\left(\mathcal{X}_{i}\right)_{k_{i}}$, condition (ii) is automatically satisfied: Indeed, if we write $h_{i} \stackrel{\text { def }}{=} \operatorname{rk}_{\mathbb{Z}}\left(H_{i}\right)$ - so $h_{i}>0$ since $\Gamma_{\left(\mathcal{X}_{i}\right)_{k_{i}}}^{\mathrm{c}}$ is assumed to be noncontractible then to assert that condition (ii) fails to hold - i.e., that there are "no new cycles in the dual graph" - is to assert that we have an equality of Euler characteristics:

$$
\left(\sum_{j} g_{Z, j}\right)+h_{i}-1=l\left\{\left(\sum_{j} g_{X, j}\right)+h_{i}-1\right\}
$$

(where the first (respectively, second) sum is the sum of the genera of the irreducible components of the geometric special fiber of $\left(\mathcal{Z}_{i}\right)_{\mathcal{O}_{K_{i}}}\left(\right.$ respectively, $\left.\left(\mathcal{X}_{i}\right)_{\mathcal{O}_{K_{i}}}\right)$ ). But, since

$$
\sum_{j}\left(g_{Z, j}-1\right)=\sum_{j} l\left(g_{X, j}-1\right)
$$

we thus conclude that $(l-1)=\left\{\sum_{j}(l-1)\right\}+h_{i}(l-1)$, hence that $1=\left(\sum_{j} 1\right)+h_{i}$ - which is absurd, since both the sum and $h_{i}$ are $\geq 1$. This completes the proof.

Remark 2.4.1. The author would like to thank A. Tamagawa for explaining to him the utility of Raynaud's theorem in this sort of situation.

In the following discussion, to keep the notation simple, we shall replace $\left(X_{i}\right)_{K_{i}}$ by some $\left(Z_{i}\right)_{K_{i}}$ as in Lemma 2.4. Thus, $V_{i}^{\text {new }}$ is a $G_{K_{i}}$-quotient module of $V_{i}$. Moreover, we have a surjection

$$
V_{i}^{\text {new }} \rightarrow\left(H_{i}^{\text {new }}\right)_{\widehat{\mathbb{Z}}}
$$

such that the quotient $\left(H_{i}\right)_{\widehat{\mathbb{Z}}} \rightarrow\left(H_{i}^{\text {new }}\right)_{\widehat{\mathbb{Z}}}$ is defined over $\mathbb{Z}$, i.e., arises from a quotient $H_{i} \rightarrow H_{i}^{\text {new }}$. (Indeed, this last assertion follows from the fact that the quotient $H_{i} \rightarrow H_{i}^{\text {new }}$ arises as the cokernel (modulo torsion) of the morphism induced on first singular cohomology modules by a finite (ramified) covering of graphs - i.e., the covering induced on dual graphs by the covering $\left(\mathcal{Z}_{i}\right)_{\mathcal{O}_{K_{i}}} \rightarrow\left(\mathcal{X}_{i}\right)_{\mathcal{O}_{K_{i}}}$ of Lemma 2.4.) 
On the other hand, the cup product on group cohomology gives rise to a nondegenerate (group-theoretic!) pairing

$$
V_{i}^{\vee} \otimes_{\widehat{\mathbb{Z}}} V_{i}^{\vee} \otimes_{\widehat{\mathbb{Z}}} \boldsymbol{\mu}_{\widehat{\mathbb{Z}}}\left(\bar{K}_{i}\right) \rightarrow M_{i} \stackrel{\text { def }}{=} H^{2}\left(\Delta_{X_{i}}, \boldsymbol{\mu}_{\widehat{\mathbb{Z}}}\left(\bar{K}_{i}\right)\right)(\cong \widehat{\mathbb{Z}})
$$

(where we think of $V_{i}^{\vee} \stackrel{\text { def }}{=} \operatorname{Hom}\left(V_{i}, \widehat{\mathbb{Z}}\right)$ as $H^{1}\left(\Delta_{X_{i}}, \widehat{\mathbb{Z}}\right)$ ), hence, by restriction to $\left(V_{i}^{\text {new }}\right)^{\vee} \hookrightarrow V_{i}^{\vee}$, a pairing

$$
\left(V_{i}^{\text {new }}\right)^{\vee} \otimes_{\widehat{\mathbb{Z}}}\left(V_{i}^{\text {new }}\right)^{\vee} \otimes_{\widehat{\mathbb{Z}}} \boldsymbol{\mu}_{\widehat{\mathbb{Z}}}\left(\bar{K}_{i}\right) \rightarrow M_{i} \stackrel{\text { def }}{=} H^{2}\left(\Delta_{X_{i}}, \boldsymbol{\mu}_{\widehat{\mathbb{Z}}}\left(\bar{K}_{i}\right)\right)(\cong \widehat{\mathbb{Z}})
$$

which is still nondegenerate (over $\mathbb{Q}$ ), since it arises from an ample line bundle - namely, the restriction of the polarization determined by the theta divisor on the Jacobian of $\left(X_{i}\right)_{K_{i}}$ to the "new part" of $\left(X_{i}\right)_{K_{i}}$. This pairing determines an "isogeny" (i.e., a morphism which is an isomorphism over $\mathbb{Q}$ ):

$$
\left(V_{i}^{\text {new }}\right)^{\vee} \otimes_{\widehat{\mathbb{Z}}} \boldsymbol{\mu}_{\widehat{\mathbb{Z}}}\left(\bar{K}_{i}\right) \otimes_{\widehat{\mathbb{Z}}} M_{i}^{\vee} \hookrightarrow V_{i}^{\text {new }}
$$

Thus, if we take the dual of the surjection discussed in the preceding paragraph, then we obtain an inclusion

$$
\left(H_{i}^{\text {new }}\right)_{\widehat{\mathbb{Z}}}^{\vee} \otimes \boldsymbol{\mu}_{\widehat{\mathbb{Z}}}\left(\bar{K}_{i}\right) \otimes M_{i}^{\vee} \hookrightarrow\left(V_{i}^{\text {new }}\right)^{\vee} \otimes \boldsymbol{\mu}_{\widehat{\mathbb{Z}}}\left(\bar{K}_{i}\right) \otimes M_{i}^{\vee} \hookrightarrow V_{i}^{\text {new }}
$$

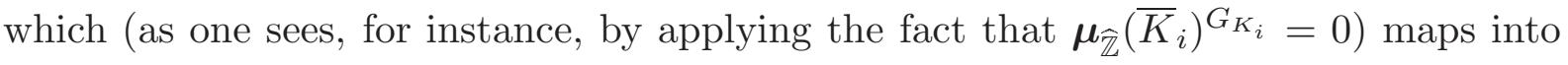
the kernel of the surjection $V_{i}^{\text {new }} \rightarrow\left(H_{i}^{\text {new }}\right)_{\widehat{\mathbb{Z}}}$.

Next, let us observe that the kernel $N_{i}$ of the surjection of unramified $G_{K_{i}}$-modules (i.e., $G_{k_{i}}$-modules)

$$
V_{i}^{\text {etl }} \rightarrow\left(H_{i}^{\text {new }}\right)_{\mathbb{Z}_{p}}
$$

satisfies:

$$
H^{0}\left(G_{k_{i}}, N_{i} \otimes \mathbb{Q}_{p}\right)=H^{1}\left(G_{k_{i}}, N_{i} \otimes \mathbb{Q}_{p}\right)=0
$$

(Indeed, $N_{i}$ arises as a submodule of the module of $p$-power torsion points of an abelian variety over $k_{i}$, so the vanishing of these cohomology groups follows from the Riemann Hypothesis for abelian varieties over finite fields (cf., e.g., [Mumf], p. 206), i.e., the fact that (some power of) the Frobenius element of $G_{k_{i}}$ acts on $N_{i}$ with eigenvalues which are algebraic numbers with complex absolute values equal to a nonzero rational power of $p$.) In particular, we conclude that the above surjection admits a unique $G_{K_{i}}$-equivariant splitting $\left(H_{i}^{\text {new }}\right)_{\mathbb{Z}_{p}} \hookrightarrow\left(V_{i}^{\text {etl }}\right)_{\mathbb{Q}_{p}}$. Similarly, (by taking Cartier duals) the injection $\left(H_{i}^{\text {new }}\right)_{\mathbb{Z}_{p}}^{\vee} \otimes \boldsymbol{\mu}_{\mathbb{Z}_{p}}\left(\bar{K}_{i}\right) \otimes M_{i}^{\vee} \hookrightarrow V_{i}^{\text {mlt }}$ also admits a unique $G_{K_{i}}$-equivariant splitting over $\mathbb{Q}_{p}$. Thus, by applying these splittings, we see that the $G_{K_{i}}$-action on $\left(V_{i}^{\text {new }}\right)_{\mathbb{Z}_{p}}$ determines a $p$-adic extension class

$$
\left(\eta_{i}\right)_{\mathbb{Z}_{p}} \in\left\{\left(H_{i}^{\text {new }}\right)_{\mathbb{Q}_{p}}^{\vee}\right\}^{\otimes 2} \otimes M_{i}^{\vee} \otimes\left(H^{1}\left(K_{i}, \boldsymbol{\mu}_{\widehat{\mathbb{Z}}}\left(\bar{K}_{i}\right)\right) / H_{f}^{1}\left(K_{i}, \boldsymbol{\mu}_{\widehat{\mathbb{Z}}}\left(\bar{K}_{i}\right)\right)\right)=\left\{\left(H_{i}^{\text {new }}\right)_{\mathbb{Q}_{p}}^{\vee}\right\}^{\otimes 2} \otimes M_{i}^{\vee}
$$


where (by Proposition 1.2.1, (vii)) $H^{1}\left(K_{i}, \boldsymbol{\mu}_{\widehat{\mathbb{Z}}}\left(\bar{K}_{i}\right)\right)$ may be identified with $\left(K_{i}^{\times}\right)^{\wedge}$, and we define

$$
H_{f}^{1}\left(K_{i}, \boldsymbol{\mu}_{\widehat{\mathbb{Z}}}\left(\bar{K}_{i}\right)\right) \stackrel{\text { def }}{=} \mathcal{O}_{K_{i}}^{\times} \subseteq\left(K_{i}^{\times}\right)^{\wedge} \stackrel{\sim}{\rightarrow} H^{1}\left(K_{i}, \boldsymbol{\mu}_{\widehat{\mathbb{Z}}}\left(\bar{K}_{i}\right)\right)
$$

so the quotient group $\left(H^{1}\left(K_{i}, \boldsymbol{\mu}_{\widehat{\mathbb{Z}}}\left(\bar{K}_{i}\right)\right) / H_{f}^{1}\left(K_{i}, \boldsymbol{\mu}_{\widehat{\mathbb{Z}}}\left(\bar{K}_{i}\right)\right)\right)$ may be identified with $\widehat{\mathbb{Z}}$.

Next, let us observe that the kernel $N_{i}^{\prime}$ of $\left(V_{i}^{\text {new }}\right)_{\widehat{\mathbb{Z}}^{\prime}} \rightarrow\left(H_{i}^{\text {new }}\right)_{\widehat{\mathbb{Z}}^{\prime}}$ is an unramified representation of $G_{K_{i}}$ (since it arises from the module of prime-to- $p$-power torsion points of a semi-abelian variety over $k_{i}$ ). Moreover, the injection of unramified $G_{K_{i}}$-modules

$$
\left(H_{i}^{\text {new }}\right)_{\widehat{\mathbb{Z}}^{\prime}}^{\vee} \otimes \boldsymbol{\mu}_{\widehat{\mathbb{Z}}^{\prime}}\left(\bar{K}_{i}\right) \otimes M_{i}^{\vee} \hookrightarrow N_{i}^{\prime}
$$

splits uniquely over $\mathbb{Q}$, since (by the Riemann Hypothesis for abelian varieties over finite fields - cf., e.g., [Mumf], p. 206) the Frobenius element of $G_{k_{i}}$ acts on the smaller module (respectively, quotient by this smaller module) with weight 1 (respectively, $\frac{1}{2}$ ). Thus, just as in the $p$-adic case, we may construct a prime-to-p-adic extension class $\left(\eta_{i}\right)_{\widehat{\mathbb{Z}}^{\prime}}$ from the $G_{K_{i}}$-action on $\left(V_{i}^{\text {new }}\right)_{\widehat{\mathbb{Z}}^{\prime}}$, which, together with $\left(\eta_{i}\right)_{\mathbb{Z}_{p}}$, yields an extension class (cf. [FC], Chapter III, Corollary 7.3):

$\eta_{i} \in\left\{\left(H_{i}^{\text {new }}\right)_{\widehat{\mathbb{Z}}}^{\vee}\right\}^{\otimes 2} \otimes M_{i}^{\vee} \otimes\left\{H^{1}\left(K_{i}, \boldsymbol{\mu}_{\widehat{\mathbb{Z}}}\left(\bar{K}_{i}\right)\right) / H_{f}^{1}\left(K_{i}, \boldsymbol{\mu}_{\widehat{\mathbb{Z}}}\left(\bar{K}_{i}\right)\right)\right\} \otimes \mathbb{Q}=\left\{\left(H_{i}^{\text {new }}\right)_{\widehat{\mathbb{Z}}}^{\vee}\right\}^{\otimes 2} \otimes M_{i}^{\vee} \otimes \mathbb{Q}$

That is to say, $\eta_{i}$ may be thought of as a (group-theoretically reconstructible!) bilinear form:

$$
\langle-,-\rangle_{i}:\left(H_{i}^{\text {new }}\right)_{\widehat{\mathbb{Z}}}^{\otimes 2} \rightarrow\left(M_{i}^{\vee}\right)_{\mathbb{Q}} \stackrel{\text { def }}{=} M_{i}^{\vee} \otimes \mathbb{Q}
$$

Moreover:

Lemma 2.5. Assume that $\left(\mathcal{X}_{i}\right)_{\mathcal{O}_{K_{i}}}$ arises as some " $\left(\mathcal{Z}_{i}\right)_{\mathcal{O}_{K_{i}}}$ " as in Lemma 2.4. Then:

(i) (Positive Rational Structures) The image of $\left(H_{i}^{\text {new }}\right)^{\otimes 2}$ under the morphism $\left(H_{i}^{\text {new }}\right)_{\widehat{\mathbb{Z}}}^{\otimes 2} \rightarrow\left(M_{i}^{\vee}\right)_{\mathbb{Q}}$ forms a rank one $\mathbb{Z}$-submodule of $\left(M_{i}^{\vee}\right)_{\mathbb{Q}}$. Moreover, for any two nonzero elements $a, b \in H_{i},\langle a, a\rangle_{i}$ differs from $\langle b, b\rangle_{i}$ by a factor in $\mathbb{Q}>0$ (i.e., a positive rational number). In particular, this image determines a "Q⿻ $>0$-structure" on $\left(M_{i}^{\vee}\right)_{\mathbb{Q}}$, i.e., a $\mathbb{Q}$-rational structure on $\left(M_{i}^{\vee}\right)_{\mathbb{Q}}$, together with a collection of generators of this $\mathbb{Q}$-rational structure that differ from one another by factors in $\mathbb{Q}>0$. Finally, this $\mathbb{Q}>0^{-}$ structure is the same as the $\mathbb{Q}_{>0}$-structure on $M_{i}^{\vee}$ determined by the first Chern class of an ample line bundle on $\left(X_{i}\right)_{K_{i}}$ in $M_{i}=H^{2}\left(\Delta_{X_{i}}, \boldsymbol{\mu}_{\widehat{\mathbb{Z}}}\left(\bar{K}_{i}\right)\right)$.

(ii) (Preservation of Degree) The isomorphism

$$
M_{1}=H^{2}\left(\Delta_{X_{1}}, \boldsymbol{\mu}_{\widehat{\mathbb{Z}}}\left(\bar{K}_{1}\right)\right) \stackrel{\sim}{\rightarrow} H^{2}\left(\Delta_{X_{2}}, \boldsymbol{\mu}_{\widehat{\mathbb{Z}}}\left(\bar{K}_{2}\right)\right)=M_{2}
$$

induced by $\alpha_{X}$ preserves the elements on both sides determined by the first Chern class of a line bundle on $\left(X_{i}\right)_{K_{i}}$ of degree 1 . 
Proof. Indeed, assertion (i), follows formally from [FC], Chapter III, Corollary 7.3, and Theorem 10.1, (iii) (by considering "new part" of the Jacobian of $\left(X_{i}\right)_{K_{i}}$ equipped with the polarization induced by the theta polarization on the Jacobian).

As for assertion (ii), the elements in question are the unique elements that, on the one hand, are rational and positive with respect to the structures discussed in assertion (i), and, on the other hand, generate $M_{i}$ as a $\widehat{\mathbb{Z}}$-module.

Remark 2.5.1. Note that the conclusion of Lemma 2.5, (ii), is valid not just for $\left(X_{i}\right)_{K_{i}}$, but for any finite étale cover of the original $\left(X_{i}\right)_{K_{i}}$, i.e., even if this cover does not arise as some " $\left(\mathcal{Z}_{i}\right)_{\mathcal{O}_{K_{i}}}$ " as in Lemma 2.4. Indeed, this follows from the fact that the crucial " $\mathbb{Q}>0$-structure" of Lemma 2.5, (i), is preserved by pull-back to such a cover, which just multiplies the Chern class at issue in Lemma 2.5, (ii), by the degree of the cover (an element of $\mathbb{Q}_{>0}$ !).

Remark 2.5.2. In the discussion of [Mzk4], $\S 9$, it was not necessary to be as careful as we were in the discussion above in constructing the $p$-adic class $\left(\eta_{i}\right)_{\mathbb{Z}_{p}}$ (i.e., " $\mu_{p}$ " in the notation of loc. cit.). This is because in loc. cit., we were working over a single $p$-adic base-field " $K$ ". In this more restricted context, the extension class $\left(\eta_{i}\right)_{\mathbb{Z}_{p}}$ may be extracted much more easily from $V_{i}$ by simply forming the quotient by the submodule of $H^{1}\left(K_{i}, \operatorname{Ker}\left(\left(V_{i}\right)_{\mathbb{Z}_{p}} \rightarrow\left(H_{i}\right)_{\mathbb{Z}_{p}}\right)\right)$ generated by the elements which are "crystalline", or, more simply, of "geometric origin" (i.e., arise from $\mathcal{O}_{K}$-rational points of the formal group associated to the $p$-divisible group determined — via "Tate's theorem" (cf. Theorem 4 of [Tate] $)$ - by the $G_{K_{i}}$-module $\left.\operatorname{Ker}\left(\left(V_{i}\right)_{\mathbb{Z}_{p}} \rightarrow\left(H_{i}\right)_{\mathbb{Z}_{p}}\right)\right)$. Unfortunately, the author omitted a detailed discussion of this aspect of the argument in the discussion of [Mzk4], $\S 9$.

Remark 2.5.3. Relative to Remark 2.5.2, we note nevertheless that even in the discussion of [Mzk4], 99 , it is still necessary to work (at least until one recovers the

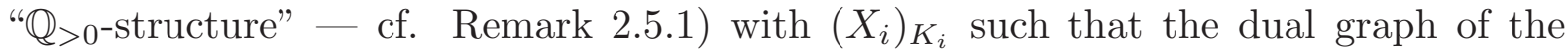
special fiber $\left(\mathcal{X}_{i}\right)_{k_{i}}$ is noncontractible. This minor technical point was omitted in the discussion of [Mzk4], $\S 9$.

Next, let us write $\left(\mathcal{X}_{i}^{\log }\right)_{\mathcal{O}_{K_{i}}}$ for the log scheme obtained by equipping $\left(\mathcal{X}_{i}\right)_{\mathcal{O}_{K_{i}}}$ with the $\log$ structure determined by the monoid of regular functions $\in \mathcal{O}_{\left(\mathcal{X}_{i}\right)_{\mathcal{O}_{K}}}$ which are invertible on the open subscheme $\left(X_{i}\right)_{K_{i}} \subseteq\left(\mathcal{X}_{i}\right)_{\mathcal{O}_{K_{i}}}$. Thus, in the terminology of [Kato2], $\left(\mathcal{X}_{i}^{\log }\right)_{\mathcal{O}_{K_{i}}}$ is log regular. Also, let us write $\left(\mathcal{X}_{i}^{\log }\right)_{k_{i}}$ for the log scheme obtained by equipping $\left(\mathcal{X}_{i}\right)_{k_{i}}$ with the log structure determined by restricting the log structure of $\left(\mathcal{X}_{i}^{\text {log }}\right)_{\mathcal{O}_{K_{i}}}$. Thus, the quotient $\Pi_{\left(X_{i}\right)_{K_{i}}} \rightarrow \Pi_{\left(X_{i}\right)_{K_{i}}}^{\text {adm }}$ determines a "universal admissible covering"

$$
\left(\widetilde{\mathcal{X}}_{i}^{\log }\right)_{k_{i}} \rightarrow\left(\mathcal{X}_{i}^{\log }\right)_{k_{i}}
$$


of $\left(\mathcal{X}_{i}^{\log }\right)_{k_{i}}$.

Now let us choose a connected component $\widetilde{\mathcal{I}}_{i}$ of the $k_{i}$-smooth locus (i.e., the complement of the nodes) of $\left(\widetilde{\mathcal{X}}_{i}^{\log }\right)_{k_{i}}$. Write $\mathcal{I}_{i} \subseteq\left(\mathcal{X}_{i}\right)_{k_{i}}$ for the image of $\widetilde{\mathcal{I}}_{i}$ in $\left(\mathcal{X}_{i}\right)_{k_{i}}$. Thus,

$$
\widetilde{\mathcal{I}}_{i} \rightarrow \mathcal{I}_{i}
$$

is a "tame universal covering" of $\mathcal{I}_{i}$ (i.e., a universal covering of the hyperbolic curve $\mathcal{I}_{i}$ among those finite étale coverings that are tamely ramified at the "cusps" of this hyperbolic curve). In the following discussion, we shall also assume, for simplicity, that $\mathcal{I}_{i}$ is geometrically connected over $k_{i}$ (a condition that may always be achieved by replacing $K_{i}$ by a finite unramified extension of $K_{i}$ ).

Now the Galois group $\Pi_{\mathcal{I}_{i}}$ of this covering may also be thought of as the quotient of the decomposition group in $\Pi_{\left(X_{i}\right)_{K_{i}}}^{\mathrm{adm}}$ of $\widetilde{\mathcal{I}}_{i}$ by its inertia group. In particular, since $\Pi_{\mathcal{I}_{i}}$ is formed by taking the quotient by this inertia group, it follows that the surjection $\Pi_{\left(X_{i}\right)_{K_{i}}}^{\mathrm{adm}} \rightarrow G_{k_{i}}^{\mathrm{log}}$ induces a natural surjection

$$
\Pi_{\mathcal{I}_{i}} \rightarrow G_{k_{i}}
$$

whose kernel is the geometric (tame) fundamental group $\pi_{1}^{\text {tame }}\left(\left(\mathcal{I}_{i}\right)_{\bar{k}_{i}}\right)$ of $\mathcal{I}_{i}$.

Finally, we observe that it makes sense to speak of $\widetilde{\mathcal{I}}_{1}$ and $\widetilde{\mathcal{I}}_{2}$ as corresponding via $\alpha_{X}$. Indeed, by Lemma 2.3, $\alpha_{X}$ induces an isomorphism between the pro-graphs determined by the $\left(\widetilde{\mathcal{X}}_{i}^{\log }\right)_{k_{i}}$. Thus, the $\widetilde{\mathcal{I}}_{i}$ may be said to correspond via $\alpha_{X}$ when the vertices that they determine in these pro-graphs correspond. Moreover, when the $\widetilde{\mathcal{I}}_{i}$ correspond via $\alpha_{X}$, it follows (by considering the stabilizer of the vertex determined by $\left.\widetilde{\mathcal{I}}_{i}\right)$ that $\alpha_{X}$ induces a bijection between the respective decomposition groups $\mathcal{D}_{i}$ in $\Pi_{\left(X_{i}\right)_{K_{i}}}^{\mathrm{adm}}$ of $\widetilde{\mathcal{I}}_{i}$, as well as between the respective inertia subgroups of these decomposition groups $\mathcal{D}_{i}$ (which may be characterized group-theoretically as the centers of the subgroups $\mathcal{D}_{i} \bigcap \operatorname{Ker}\left(\Pi_{\left(X_{i}\right)_{K_{i}}}^{\text {adm }} \rightarrow G_{k_{i}}\right)$, since $\pi_{1}^{\text {tame }}\left(\left(\mathcal{I}_{i}\right)_{\bar{k}_{i}}\right)$ is center-free - cf. Lemma 1.3.10). Thus, in summary, $\alpha_{X}$ induces a commutative diagram:

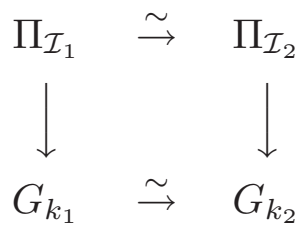

We are now ready (cf. [Mzk4], §7) to apply the main result of [Tama1]. This result states that commutative diagrams as above are in natural bijective correspondence with commutative diagrams

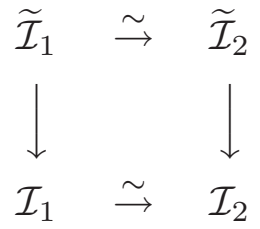


lying over commutative diagrams

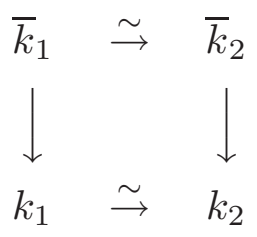

(cf. Theorem 1.3.11). In particular, these commutative diagrams induce an isomorphism

$$
\left(\widehat{\mathbb{Z}}^{\prime} \cong\right) H_{\mathrm{c}}^{2}\left(\left(\mathcal{I}_{1}\right)_{\bar{k}_{1}}, \boldsymbol{\mu}_{\widehat{\mathbb{Z}}^{\prime}}\left(\bar{k}_{1}\right)\right) \stackrel{\sim}{\rightarrow} H_{\mathrm{c}}^{2}\left(\left(\mathcal{I}_{2}\right)_{\bar{k}_{2}}, \boldsymbol{\mu}_{\widehat{\mathbb{Z}}^{\prime}}\left(\bar{k}_{2}\right)\right)\left(\cong \widehat{\mathbb{Z}}^{\prime}\right)
$$

(where " $H_{\mathrm{c}}^{2}$ " denotes étale cohomology with compact supports — cf. [Milne], Chapter III, Proposition 1.29; Remark 1.30) which maps the element "1" (i.e., the element determined by the first Chern class of a line bundle of degree 1) on the left to the element " 1 " on the right. (Indeed, this follows from the fact that the morphism $\mathcal{I}_{1} \stackrel{\sim}{\rightarrow} \mathcal{I}_{2}$ appearing in the above commutative diagram is an isomorphism, hence of degree 1.) Note that the isomorphism $\boldsymbol{\mu}_{\widehat{\mathbb{Z}}^{\prime}}\left(\bar{k}_{1}\right) \stackrel{\sim}{\rightarrow} \boldsymbol{\mu}_{\widehat{\mathbb{Z}}^{\prime}}\left(\bar{k}_{2}\right)$ that we use here is that obtained from the commutative diagram above, i.e., that provided by Theorem 1.3.11.

Lemma 2.6. (Compatibility of Isomorphisms Between Roots of Unity) Assume that $\left(\mathcal{X}_{i}\right)_{\mathcal{O}_{K_{i}}}$ arises as some " $\left(\mathcal{Z}_{i}\right)_{\mathcal{O}_{K_{i}}}$ " as in Lemma 2.4. Then the following diagram

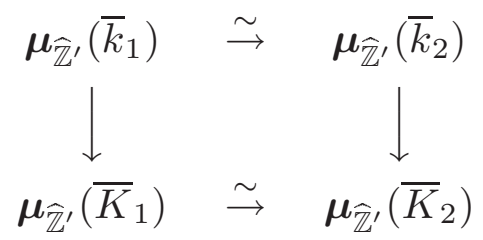

- in which the vertical morphisms are the natural ones (obtained by considering Teichmüller representatives); the upper horizontal morphism is the morphism determined by Theorem 1.3.11; and the lower horizontal morphism is the morphism determined by Proposition 1.2.1, (vi) - commutes.

Proof. Indeed, the diagram in the statement of Lemma 2.6 induces a diagram:

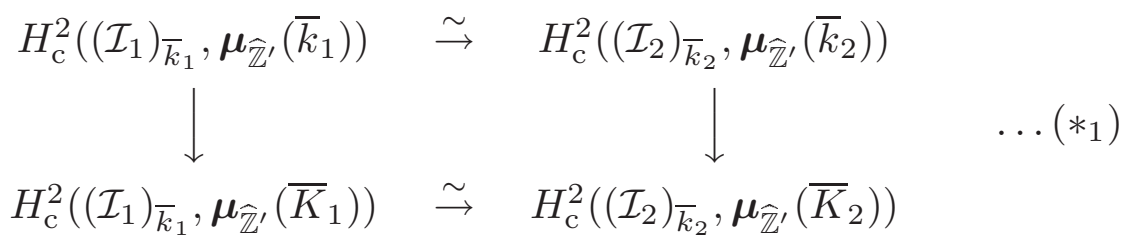

Moreover, we have a diagram

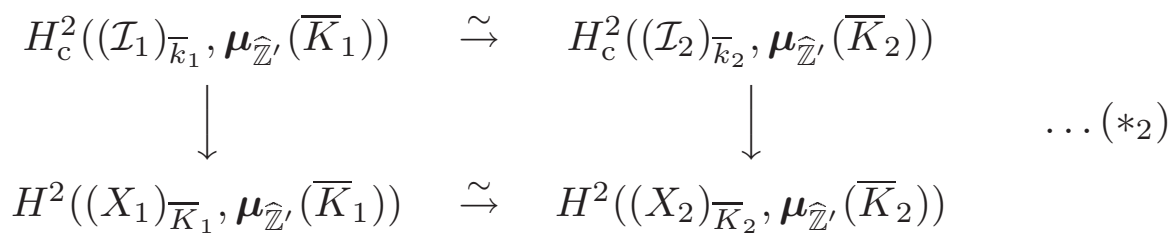


where the horizontal morphisms are induced by $\alpha_{K}$ (cf. Proposition 1.2.1, (vi)), and the vertical morphisms are induced "group-theoretically" as follows: First, observe that [since we continue to operate under the assumption ( $\left.{ }^{\mathrm{CSSN}}\right)$ ]

$$
H^{2}\left(\left(X_{i}\right)_{\bar{K}_{i}}, \boldsymbol{\mu}_{\widehat{\mathbb{Z}}^{\prime}}\left(\bar{K}_{i}\right)\right) \cong H^{2}\left(\Delta_{X_{i}}, \boldsymbol{\mu}_{\widehat{\mathbb{Z}}^{\prime}}\left(\bar{K}_{i}\right)\right) \cong H^{2}\left(\Delta_{X_{i}}^{\mathrm{adm}}, \boldsymbol{\mu}_{\widehat{\mathbb{Z}}^{\prime}}\left(\bar{K}_{i}\right)\right)
$$

while

$$
H_{\mathrm{c}}^{2}\left(\left(\mathcal{I}_{i}\right)_{\bar{k}_{i}}, \boldsymbol{\mu}_{\widehat{\mathbb{Z}}^{\prime}}\left(\bar{K}_{i}\right)\right) \cong H^{2}\left(\left(\overline{\mathcal{I}}_{i}\right)_{\bar{k}_{i}}, \boldsymbol{\mu}_{\widehat{\mathbb{Z}}^{\prime}}\left(\bar{K}_{i}\right)\right) \cong H^{2}\left(\pi_{1}\left(\left(\overline{\mathcal{I}}_{i}\right)_{\bar{k}_{i}}\right), \boldsymbol{\mu}_{\widehat{\mathbb{Z}}^{\prime}}\left(\bar{K}_{i}\right)\right)
$$

(where we write $\overline{\mathcal{I}}_{i}$ for the unique nonsingular compactification of $\mathcal{I}_{i}$ ). Moreover, since we continue to operate under the assumption $\left(*^{\mathrm{CSSN}}\right)$, it follows (cf. the discussion of "Second Cohomology Groups" in the Appendix) that the natural "push-forward" morphism in étale cohomology

$$
\begin{aligned}
\left(H^{2}\left(\pi_{1}\left(\left(\overline{\mathcal{I}}_{i}\right)_{\bar{k}_{i}}\right), \boldsymbol{\mu}_{\widehat{\mathbb{Z}}^{\prime}}\left(\bar{K}_{i}\right)\right) \cong\right) & H_{\mathrm{c}}^{2}\left(\left(\mathcal{I}_{i}\right)_{\bar{k}_{i}}, \boldsymbol{\mu}_{\widehat{\mathbb{Z}}^{\prime}}\left(\bar{K}_{i}\right)\right) \\
& \rightarrow H^{2}\left(\left(\mathcal{X}_{i}\right)_{k_{i}} \otimes_{k_{i}} \bar{k}_{i}, \boldsymbol{\mu}_{\widehat{\mathbb{Z}}^{\prime}}\left(\bar{K}_{i}\right)\right)\left(\cong H^{2}\left(\Delta_{X_{i}}^{\mathrm{et}}, \boldsymbol{\mu}_{\widehat{\mathbb{Z}}^{\prime}}\left(\bar{K}_{i}\right)\right)\right)
\end{aligned}
$$

may be reconstructed group-theoretically (by using the various natural homomorphisms $\pi_{1}\left(\left(\overline{\mathcal{I}}_{i}\right)_{\bar{k}_{i}}\right) \rightarrow \Delta_{X_{i}}^{\text {et }}$ [well-defined up to composite with an inner automorphism]). Thus, the desired vertical morphisms of diagram $\left(*_{2}\right)$ may be obtained by composing these "push-forward" morphisms with the morphisms

$$
H^{2}\left(\Delta_{X_{i}}^{\mathrm{et}}, \boldsymbol{\mu}_{\widehat{\mathbb{Z}}^{\prime}}\left(\bar{K}_{i}\right)\right) \rightarrow H^{2}\left(\Delta_{X_{i}}^{\mathrm{adm}}, \boldsymbol{\mu}_{\widehat{\mathbb{Z}}^{\prime}}\left(\bar{K}_{i}\right)\right)
$$

induced by the surjections $\Delta_{X_{i}}^{\mathrm{adm}} \rightarrow \Delta_{X_{i}}^{\text {et }}$ [which are group-theoretic by Lemma 2.2 , (ii)]. In particular, we thus see that the vertical morphisms of diagram $\left(*_{2}\right)$ are grouptheoretic, i.e., (in other words) diagram $\left(*_{2}\right)$ commutes.

Now let us compose the above two diagrams $\left(*_{1}\right),\left(*_{2}\right)$ to form a single diagram:

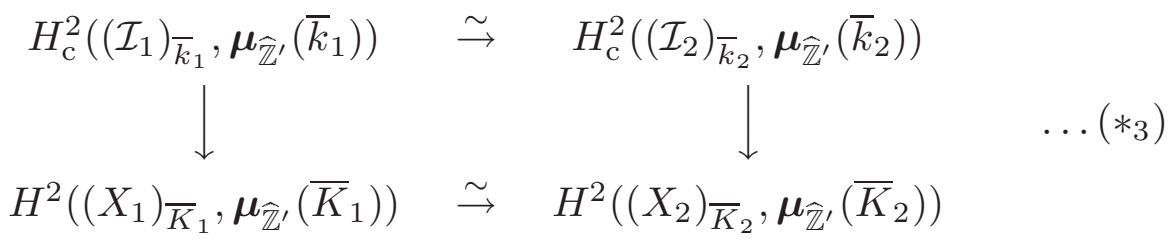

Note that this diagram $\left(*_{3}\right)$ commutes, since, by Lemma 2.5, (ii) [applied to the lower horizontal morphism of $\left.\left(*_{3}\right)\right]$; the discussion immediately preceding the present Lemma 2.6 [applied to the upper horizontal morphism of $\left(*_{3}\right)$ ]; and the discussion of "Second Cohomology Groups" in the Appendix [applied to the vertical morphisms of $\left(*_{3}\right)$ ], all of the morphisms of this diagram are compatible with the elements " 1 " determined by the first Chern class of a line bundle of degree 1. But this implies that diagram $\left(*_{1}\right)$ commutes (since diagram $\left(*_{2}\right)$ has already been shown to be commutative, and all the arrows in both of these diagrams $\left(*_{1}\right),\left(*_{2}\right)$ are isomorphisms between rank one 
free $\widehat{\mathbb{Z}}^{\prime}$-modules). On the other hand, since diagram $\left(*_{1}\right)$ was obtained by applying the functors $H_{\mathrm{c}}^{2}\left(\left(\mathcal{I}_{i}\right)_{\bar{k}_{i}},-\right)$ (which are manifestly faithful, at least when restricted to constant coefficient modules which are finitely generated and free over $\widehat{\mathbb{Z}}^{\prime}$ - as in the present situation) to the diagram appearing in the statement of Lemma 2.6, we thus conclude that the diagram appearing in the statement of Lemma 2.6 is commutative, as desired.

The significance of Lemma 2.6 from our point of view is the following: Lemma 2.6 implies that $\alpha_{K}$ induces an isomorphism

$$
\alpha_{G_{k} \log }: G_{k_{1}}^{\log } \stackrel{\sim}{\rightarrow} G_{k_{2}}^{\log }
$$

which satisfies the hypothesis of Proposition 1.2.4, (iii). Thus, we conclude from Proposition 1.2.4, (iii), that $\alpha_{G_{k}^{\log }}$ arises geometrically. In particular, it follows that we may apply [Mzk4], Theorem 7.2, to the commutative diagram

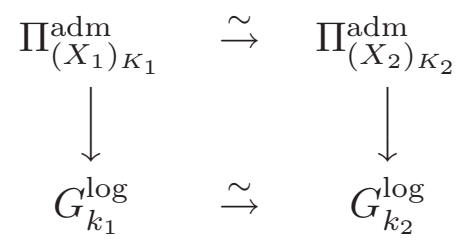

(where we note that Lemma 2.6 also implies — when translated into the terminology of [Mzk4], $\S 7$ - that the "RT-degree" associated to this commutative diagram is 1, as is necessary for the application of [Mzk4], Theorem 7.2). In particular, we conclude that the above commutative diagram of fundamental groups arises geometrically from a commutative diagram:

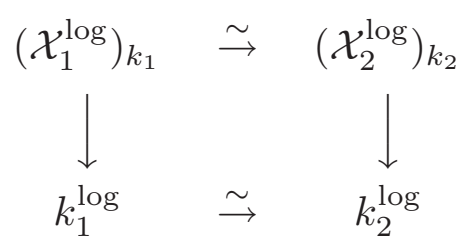

Moreover, the isomorphism exhibited in the upper horizontal arrow of this commutative diagram is easily seen to be functorial with respect to arbitrary finite étale coverings of the $\left(X_{i}\right)_{K_{i}}$ (i.e., not just coverings that arise from finite étale coverings of the $\left.\left(\mathcal{X}_{i}\right)_{\mathcal{O}_{K_{i}}}\right)$. Indeed, this functoriality follows formally from the uniqueness assertion in Proposition 1.2.4, (iii), and the fact that dominant (i.e., not just finite étale) morphisms between proper hyperbolic curves in characteristic $p$ may be distinguished by considering the morphisms that they induce between the respective Jacobians, hence, in particular, by the morphisms that they induce between the $l$-power torsion points (where $l \neq p$ ) of the respective Jacobians. [Note also that the morphisms between log structures at the nodes are uniquely determined by the morphisms between the various normal bundles on the branches at the nodes - cf. [Mzk3], §3.7, 3.8, 3.10.] Thus, in summary: 
Theorem 2.7. (Group-Theoretic Reconstruction of the Logarithmic Special Fiber of a $\boldsymbol{p}$-adic Hyperbolic Curve) Let $p$ be a prime number. For $i=1,2$, let $K_{i}$ be a finite extension of $\mathbb{Q}_{p}$, and $\left(X_{i}\right)_{K_{i}}$ a hyperbolic curve over $K_{i}$ whose associated pointed stable curve has stable reduction over $\mathcal{O}_{K_{i}}$. Denote the resulting "stable model" of $\left(X_{i}\right)_{K_{i}}$ over $\mathcal{O}_{K_{i}}$ by $\left(\mathcal{X}_{i}\right)_{\mathcal{O}_{K_{i}}}$. Assume that we have chosen basepoints of the $\left(X_{i}\right)_{K_{i}}$ (which thus induce basepoints of the $K_{i}$ ). Then every isomorphism of profinite groups $\Pi_{\left(X_{1}\right)_{K_{1}}} \stackrel{\sim}{\rightarrow} \Pi_{\left(X_{2}\right)_{K_{2}}}$ induces commutative diagrams:

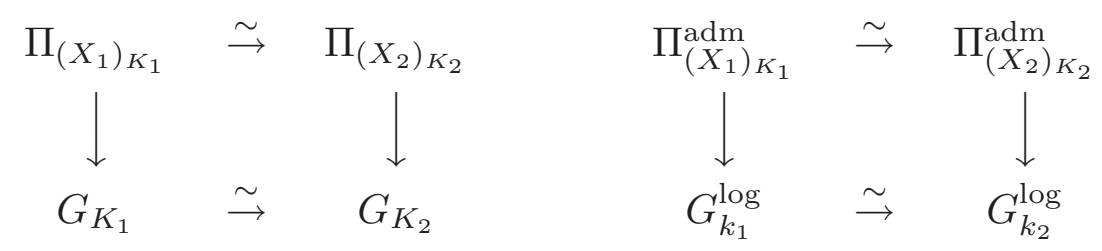

Moreover, the latter commutative diagram (of admissible quotients $\Pi_{\left(X_{i}\right)_{K_{i}}}^{\mathrm{adm}}$ of the

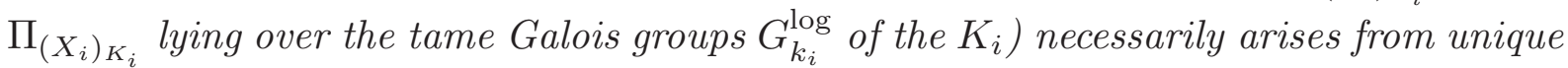
commutative diagrams of log schemes

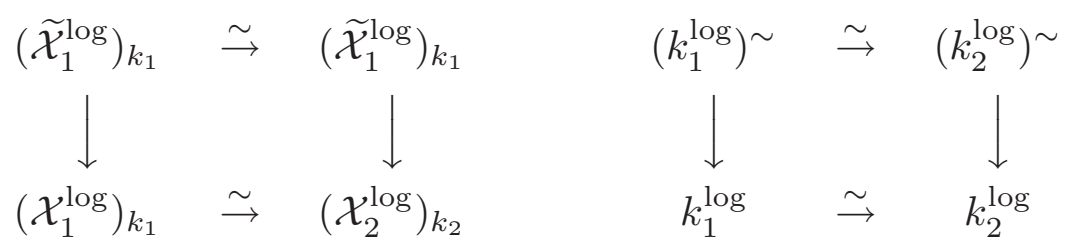

where the commutative diagram on the left lies over the commutative diagram on the right (which is as in Proposition 1.2.4, (iii)). Here, we equip $\operatorname{Spec}\left(\mathcal{O}_{K_{i}}\right)$ (respectively, $\left.\operatorname{Spec}\left(k_{i}\right) ;\left(\mathcal{X}_{i}\right)_{\mathcal{O}_{K_{i}}} ;\left(\mathcal{X}_{i}\right)_{k_{i}}\right)$ with the log structure determined by the closed point (respectively, determined by restricting the log structure on $\operatorname{Spec}\left(\mathcal{O}_{K_{i}}\right)$; determined by the monoid of functions invertible on the open subscheme $\left(X_{i}\right)_{K_{i}}$; determined by restricting the log structure on $\left(\mathcal{X}_{i}\right)_{\mathcal{O}_{K_{i}}}$ ) and denote the resulting log scheme by $\mathcal{O}_{K_{i}}^{\log }$ (respectively, $\left.k_{i}^{\log } ;\left(\mathcal{X}_{i}^{\log }\right)_{\mathcal{O}_{K_{i}}} ;\left(\mathcal{X}_{i}^{\log }\right)_{k_{i}}\right)$; the vertical morphisms in the above commutative diagrams of log schemes are the universal coverings induced by the various basepoints chosen.

Proof. First, note that the additional assumptions that were used in the course of the above discussion - e.g., "( $\left.{ }^{\mathrm{CSSN}}\right)$ "; the assumption that $\left(\mathcal{X}_{i}\right)_{\mathcal{O}_{K_{i}}}$ arise as some " $\left(\mathcal{Z}_{i}\right)_{\mathcal{O}_{K_{i}}}$ " as in Lemma 2.4 - were applied only to show that the hypotheses of Proposition 1.2.4, (iii) (and [Mzk4], Theorem 7.2) are satisfied. Moreover, we observe that although [Mzk4], Theorem 7.2, is only stated in the proper singular case, it extends immediately to the affine singular, as well as affine nonsingular, cases. Thus, (cf. Remark 2.5.1; the discussion surrounding " $\left(*^{\mathrm{CSSN}}\right)$ ") one concludes that - except when $\left(X_{i}\right)_{K_{i}}$ is proper, with good reduction - one may reconstruct the logarithmic special fiber in a functorial fashion (i.e., with respect to finite étale coverings of the $\left(X_{i}\right)_{K_{i}}$ ), as desired.

In the case that $\left(X_{i}\right)_{K_{i}}$ is proper, but has good reduction over $\mathcal{O}_{K_{i}}$, we may still reconstruct its logarithmic special fiber (despite the fact that [Tama1], Theorem 4.3, 
is only stated in [Tama1] for affine hyperbolic curves!) by arguing as follows: First of all, we observe that in the case of good reduction, the log structure of the special fiber of the curve is obtained by simply pulling back the log structure of $k_{i}^{\log }$. Thus, it suffices to construct the (non-logarithmic, scheme-theoretic) special fiber. Next, we observe that (after possibly enlarging $K_{i}$ ) there exist — cf., e.g., [Mzk4], the first two paragraphs of the proof of Theorem 9.2 - corresponding finite Galois étale coverings $\left(Z_{i}\right)_{K_{i}} \rightarrow\left(X_{i}\right)_{K_{i}}$ (for $\left.i=1,2\right)$, where $\left(Z_{i}\right)_{K_{i}}$ is a hyperbolic curve over $K_{i}$ with bad stable reduction $\left(\mathcal{Z}_{i}\right)_{\mathcal{O}_{K_{i}}}$ over $\mathcal{O}_{K_{i}}$. Thus, by applying Theorem 2.7 to $\left(Z_{i}\right)_{K_{i}}$ allows us to reconstruct the logarithmic special fiber $\left(\mathcal{Z}_{i}^{\log }\right)_{k_{i}}$, together with the action of the Galois group $G_{i} \stackrel{\text { def }}{=} \operatorname{Gal}\left(\left(Z_{i}\right)_{K_{i}} /\left(X_{i}\right)_{K_{i}}\right)$. Note that irreducible components of $\left(\mathcal{Z}_{i}\right)_{k_{i}}$ that dominate $\left(\mathcal{X}_{i}\right)_{k_{i}}$ may be distinguished (group-theoretically!) by the fact that their geometric fundamental groups map surjectively onto open subgroups of the geometric fundamental group of $\left(\mathcal{X}_{i}\right)_{k_{i}}$. Let us choose corresponding (closed, proper) irreducible components

$$
C_{i} \subseteq\left(\mathcal{Z}_{i}\right)_{k_{i}}
$$

that dominate (hence surject onto) $\left(\mathcal{X}_{i}\right)_{k_{i}}$. Denote the decomposition (respectively, inertia) group associated to $C_{i}$ by $D_{i} \subseteq G_{i}$ (respectively, $I_{i} \subseteq D_{i} \subseteq G_{i}$ ). Thus, $D_{i} / I_{i}$ acts faithfully on $C_{i}$, and the order $\left|I_{i}\right|$ of $I_{i}$ is a power of $p$, equal to the degree of inseparability of the function field of $C_{i}$ over the function field of $\left(\mathcal{X}_{i}\right)_{k_{i}}$. Then we may reconstruct $\left(\mathcal{X}_{i}\right)_{k_{i}}$ as a finite flat quotient of $C_{i}$ by considering the subsheaf

$$
\left(\mathcal{O}_{C_{i}}^{\left|I_{i}\right|}\right)^{D_{i}} \subseteq \mathcal{O}_{C_{i}}
$$

(i.e., the $D_{i}$-invariants of the subalgebra $\mathcal{O}_{C_{i}}^{\left|I_{i}\right|} \subseteq \mathcal{O}_{C_{i}}$, where we use that $\left|I_{i}\right|$ is a power of $p$ ). By applying the functoriality with respect to finite étale coverings of $\left(X_{i}\right)_{K_{i}}$ observed in the discussion immediately preceding the statement of Theorem 2.7, we conclude that this construction of $\left(\mathcal{X}_{i}\right)_{k_{i}}$ is independent of the choice of $\left(Z_{i}\right)_{K_{i}}, C_{i}$, and itself functorial with respect to finite étale coverings of $\left(X_{i}\right)_{K_{i}}$.

This completes our reconstruction of the logarithmic special fibers of the $\left(X_{i}\right)_{K_{i}}$, in a fashion that is functorial with respect to finite étale coverings of the $\left(X_{i}\right)_{K_{i}}$. Thus, we conclude, in particular, (from this functoriality, applied to covering transformations; the slimness of Lemma 2.2, (i)) that the morphism induced on admissible fundamental groups by the isomorphism constructed between logarithmic special fibers coincides with the original given morphism between admissible fundamental groups. This completes the proof of Theorem 2.7.

Remark 2.7.1. Given data as in Theorem 2.7, one may consider the outer Galois representation

$$
G_{K_{i}} \rightarrow \operatorname{Out}\left(\Delta_{X_{i}}\right)
$$


which is known to be injective if $r_{i}>0$ (cf. Theorem 1.3.6). Thus, at least in the case $r_{i}>0$, it is natural to ask:

What is the commensurator of $\operatorname{Im}\left(G_{K_{i}}\right)$ in $\operatorname{Out}\left(\Delta_{X_{i}}\right)$ ?

Although Theorem 2.7 does not give a complete explicit answer to this question, it tells us that, at any rate, elements of this commensurator (which define isomorphisms of the sort that are treated in Theorem 2.7) preserve the logarithmic special fiber. In particular, (although one does not know whether or not elements of this commensurator induce "self-isogenies" of $K_{i}$, i.e., are "geometric") one obtains that elements of this commensurator do induce "self-isogenies" of $k_{i}^{\log }$. [Here, by a "self-isogeny of an object", we mean an isomorphism between two finite étale coverings of the object.] Moreover, since it follows from Theorem A of [Mzk6] (cf. Theorem 1.3.4, Remark 1.3.6.2) that the centralizer of $\operatorname{Im}\left(G_{K_{i}}\right)$ in $\operatorname{Out}\left(\Delta_{X_{i}}\right)$ consists precisely of those (finitely many) automorphisms that arise geometrically (i.e., from automorphisms of $\left(X_{i}\right)_{K_{i}}$ ), it follows that a "self-isogeny" of $G_{K_{i}}$ induced by an element of this commensurator corresponds to (up to finitely many well-understood possibilities) an essentially unique element of this commensurator. This motivates the point of view that:

The "self-isogenies" of $G_{K_{i}}$ defined by elements of this commensurator which we shall refer to as quasi-conformal self-isogenies of $G_{K_{i}}$ - are natural objects to study in their own right.

The reason for the choice of the terminology "quasi-conformal" is that those selfisogenies that are "of geometric origin" — i.e., "conformal" — are (by the main theorem of [Mzk5]) precisely those which preserve the higher ramification filtration, which is closely related to the "canonical p-adic metric" on the local field in question. Thus, quasi-conformal self-isogenies do not preserve the "metric (or conformal) structure" but do preserve the "logarithmic special fiber" which one may think of as a sort of $p$-adic analogue of the "topological type" of the objects in question.

Remark 2.7.2. Note that isomorphisms

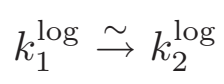

(such as those arising from "quasi-conformal isomorphisms" $G_{K_{1}} \stackrel{\sim}{\rightarrow} G_{K_{2}}$ as in Theorem 2.7) need not be "geometric" from the point of view of characteristic zero (i.e., induced by an isomorphism of fields $K_{1} \stackrel{\sim}{\rightarrow} K_{2}$ ). For instance, such an isomorphism might take the section of the log structure corresponding to $p$ to some multiple of this section by a root of unity (a situation which could never occur if the isomorphism arose from an isomorphism $K_{1} \stackrel{\sim}{\rightarrow} K_{2}$ ). Whether or not, however, this sort of phenomenon actually 
takes place in the case of "quasi-conformal isomorphisms" as in Theorem 2.7 is not clear to the author at the time of writing.

Remark 2.7.3. The theory of the present $\oint$ prompts the question:

Do isomorphisms $\Pi_{\left(X_{1}\right)_{K_{1}}} \stackrel{\sim}{\rightarrow} \Pi_{\left(X_{2}\right)_{K_{2}}}$ as in Theorem 2.7 only preserve the logarithmic special fiber or do they preserve other information as well concerning the liftings $\left(X_{i}\right)_{K_{i}}$ of the respective logarithmic special fibers?

Although the author is unable to give a complete answer to this question at the time of writing, it does appear that when the lifting in question is in some sense "canonical", then this canonicality is preserved by isomorphisms as in Theorem 2.7. In a future paper, we hope to discuss this sort of phenomenon - which may be observed, for instance, in the following cases:

(1) Serre-Tate canonical liftings;

(2) "arithmetic hyperbolic curves", i.e., hyperbolic curves isogenous to a Shimura curve [that is to say, curves which admit a finite étale covering which is isomorphic to a finite étale covering of some Shimura curve];

(3) canonical liftings in the sense of "p-adic Teichmüller theory" (cf. [Mzk1], [Mzk2])

- in more detail. Perhaps this phenomenon should be regarded as a natural extension of the phenomenon of preservation of the logarithmic fiber in the sense that canonical liftings are, in some sense, liftings that are "defined over $\mathbb{F}_{1}$ " - i.e., a hypothetical (but, of course, fictional!) absolute field of constants sitting inside $\mathbb{Z}_{p}$.

\section{Appendix: Terminology of Graph Theory}

\section{The Notion of a Semi-Graph:}

We shall refer to as a semi-graph $\Gamma$ the following collection of data:

(1) a set $\mathcal{V}$ - whose elements we refer to as "vertices";

(2) a set $\mathcal{E}$ - whose elements we refer to as "edges" - each of whose elements $e$ is a set of cardinality 2 satisfying the property " $e \neq e^{\prime} \in \mathcal{E} \Longrightarrow e \bigcap e^{\prime}=\emptyset "$;

(3) a collection $\zeta$ of maps $\zeta_{e}$ [one for each edge $\left.e\right]$ — which we refer to as the "coincidence maps" - such that $\zeta_{e}: e \rightarrow \mathcal{V} \cup\{\mathcal{V}\}$ [where we note that $\mathcal{V} \cap\{\mathcal{V}\}=\emptyset$ since $\mathcal{V} \notin \mathcal{V}]$ is a map from the set $e$ to the set $\mathcal{V} \bigcup\{\mathcal{V}\}$. 
We shall refer to the subset $\zeta_{e}^{-1}(\mathcal{V}) \subseteq e$ [i.e., the inverse image of the subset $\mathcal{V} \subseteq \mathcal{V} \bigcup\{\mathcal{V}\}$ of elements $\neq \mathcal{V}]$ as the verticial portion of an edge $e$; to the restriction of $\zeta_{e}$ to the verticial portion of $e$ as the verticial restriction of $\zeta_{e}$; and to the cardinality of the verticial portion of $e$ as the verticial cardinality of $e$. A graph $\Gamma$ is a semi-graph $\Gamma$ for which every $e \in \mathcal{E}$ has verticial cardinality precisely 2 . We will say that a graph or semigraph is finite if its sets of vertices and edges are finite. We shall refer to an element $b \in e$ as a branch of the edge $e$.

Let $\Gamma=\{\mathcal{V}, \mathcal{E}, \zeta\}$ be a semi-graph. If $e \in \mathcal{E}$ is an edge of $\Gamma$ of verticial cardinality 2 whose image via $\zeta_{e}$ consists of two (not necessarily distinct) elements $v_{1}, v_{2}$ of $\mathcal{V}$, then we shall say that $e$ joins $v_{1}$ to $v_{2}$. If $v$ is any vertex in the image of $\zeta_{e}$, then we shall say that $e$ meets or abuts to $v$. Thus, an edge of a graph always abuts to at least one vertex, while an edge of a semi-graph may abut to no vertices at all.

By thinking of vertices as points and edges as line segments that join points to points or are "open" at one or both ends, we may think of semi-graphs as defining topological spaces. Thus, it makes sense to speak of a semi-graph as being contractible (in the sense of algebraic topology). Such a semi-graph will be referred to as a tree.

Finally, a morphism between semi-graphs

$$
\Gamma=\{\mathcal{V}, \mathcal{E}, \zeta\} \rightarrow \Gamma^{\prime}=\left\{\mathcal{V}^{\prime}, \mathcal{E}^{\prime}, \zeta^{\prime}\right\}
$$

is a collection of maps $\mathcal{V} \rightarrow \mathcal{V}^{\prime} ; \mathcal{E} \rightarrow \mathcal{E}^{\prime}$; and for each $e \in \mathcal{E}$ mapping to $e^{\prime}$, an injection $e \hookrightarrow e^{\prime}$ - all of which are compatible with the verticial restrictions of the respective coincidence maps. Thus, here, we allow an edge that abuts to no (respectively, precisely one) vertex to map to an edge that abuts to any number $\geq 0$ (respectively, $\geq 1$ ) of vertices.

\section{Semi-Graphs of Profinite Groups:}

We shall refer to the following data $G$ :

(i) a finite semi-graph $\Gamma$;

(ii) for each vertex $v$ of $\Gamma$, a profinite group $G_{v}$;

(iii) for each edge $e$ of $\Gamma$, a profinite group $G_{e}$, together with, for each branch $b \in e$ mapping to a vertex $v$ via $\zeta_{e}$, a continuous homomorphism $b_{*}: G_{e} \rightarrow G_{v}$

as a semi-graph of profinite groups. When $\Gamma$ is a graph, we shall refer to this data $G$ as a graph of profinite groups.

Suppose that we are given a semi-graph of profinite groups $G$. Then to $G$, one may associate (in a natural, functorial fashion) a profinite group $\Pi_{G}$ - namely, the 
profinite analogue of the well-known construction of the fundamental group associated to a (semi-)graph of groups (cf. [Serre1], I, §5.1). One way to describe this profinite analogue is via Galois categories (cf., e.g., [SGA1] for an exposition of the theory of Galois categories) as follows: If $H$ is an arbitrary profinite group, then let us write

$$
\mathcal{B}(H)
$$

for the category of finite sets with continuous $H$-action. Then $H$ may be recovered as the "fundamental group" of the Galois category $\mathcal{B}(H)$. Also, we note that any continuous homomorphism of profinite groups $H \rightarrow H^{\prime}$ determines a natural functor $\mathcal{B}\left(H^{\prime}\right) \rightarrow \mathcal{B}(H)$ [in the reverse direction]. In particular, the homomorphisms $b_{*}$ determine functors:

$$
b^{*}: \mathcal{C}_{v} \stackrel{\text { def }}{=} \mathcal{B}\left(G_{v}\right) \rightarrow \mathcal{C}_{e} \stackrel{\text { def }}{=} \mathcal{B}\left(G_{e}\right)
$$

At any rate, to define $\Pi_{G}$, it suffices to define a Galois category $\mathcal{C}_{G}$ [which we would like to think of as " $\mathcal{B}\left(\Pi_{G}\right)$ "]. We define $\mathcal{C}_{G}$ to be the category of collections of data:

$$
\left\{S_{v} ; \alpha_{e}\right\}
$$

Here, "v" (respectively, "e") ranges over the vertices (respectively, edges of verticial cardinality 2) of $\Gamma ; S_{v}$ is an object of $\mathcal{C}_{v}$; and $\alpha_{e}: b_{1}^{*}\left(S_{v_{1}}\right) \cong b_{2}^{*}\left(S_{v_{2}}\right)$ (where $e=\left\{b_{1}, b_{2}\right\}$; $v_{i} \stackrel{\text { def }}{=} \zeta_{e}\left(b_{i}\right)$, for $\left.i=1,2\right)$ is an isomorphism in $\mathcal{C}_{e}$. Morphisms between such collections of data are defined in the evident way. One then verifies easily that this category $\mathcal{C}_{G}$ is indeed a Galois category, as desired.

\section{Pointed Stable Curves:}

Let $k$ be an algebraically closed field. Let $g, r \geq 0$ be integers such that $2 g-2+r>0$. Let $(\bar{X} \rightarrow \operatorname{Spec}(k), D \subseteq \bar{X})$ be an r-pointed stable curve of genus $g$ (where $D \subseteq \bar{X}$ is the divisor of marked points) over $k$, and set:

$$
X \stackrel{\text { def }}{=} \bar{X} \backslash D
$$

Also, let us write $X^{\prime} \subseteq X$ for the complement of the nodes in $X$.

Next, we define the dual graph

$$
\Gamma_{X}
$$

of $X$. The set of vertices of $\Gamma_{X}$ is the set of irreducible components of $X$. To avoid confusion, we shall write $X_{v}$ for the irreducible component corresponding to a vertex $v$. The set of edges of $\Gamma_{X}$ is the set of nodes of $X$. To avoid confusion, we shall write $\nu_{e}$ for the node corresponding to an edge $e$. The node $\nu_{e}$ has two branches $\nu_{e}[1]$ and $\nu_{e}[2]$, each of which lies on some well-defined irreducible component of $X$. We take 
$e \stackrel{\text { def }}{=}\left\{\nu_{e}[1], \nu_{e}[2]\right\}$ [so $e$ is of verticial cardinality 2], and let $\zeta_{e}$ be the map that sends $\nu_{e}[i]$ to the irreducible component on which the branch $\nu_{e}[i]$ lies.

Let us write $X_{v}^{\prime} \stackrel{\text { def }}{=} X_{v} \cap X^{\prime} \subseteq X$, and (for $i=1,2$ ) $X_{\nu_{e}[i]}^{\prime}$ for the scheme-theoretic intersection with $X^{\prime}$ of the completion of the branch $\nu_{e}[i]$ at the node $\nu_{e}$. Thus, $X_{\nu_{e}[i]}^{\prime}$ is noncanonically isomorphic to $\operatorname{Spec}\left(k[[t]]\left[t^{-1}\right]\right)$ (where $t$ is an indeterminate).

In the following discussion, we would like to fix ( $k$-linear) isomorphisms:

$$
X_{\nu_{e}[1]}^{\prime} \cong X_{\nu_{e}[2]}^{\prime}
$$

via which we shall identify $X_{\nu_{e}[1]}^{\prime}$ with $X_{\nu_{e}[2]}^{\prime}$ and denote the resulting object by $X_{e}^{\prime}$. In particular, we have natural morphisms $X_{e}^{\prime} \rightarrow X_{v_{1}}^{\prime}, X_{e}^{\prime} \rightarrow X_{v_{2}}^{\prime}\left(\right.$ where $v_{i} \stackrel{\text { def }}{=} \zeta_{e}\left(\nu_{e}[i]\right)$, for $i=1,2)$. One verifies immediately that the induced morphism on tame algebraic fundamental groups [i.e., corresponding to coverings which are tamely ramified at the nodes and marked points] is independent of the choice of isomorphism. Thus, the dual graph $\Gamma_{X}$, together with the result of applying " $\pi_{1}^{\text {tame }}(-)$ " [more precisely: the tame algebraic fundamental group functor, together with choices of basepoints for the various $X_{v}^{\prime}, X_{e}^{\prime}$, and choices of paths to relate these basepoints via the natural morphisms $X_{e}^{\prime} \rightarrow X_{v_{i}}^{\prime}$ discussed above] to the data $\left\{X_{v}^{\prime} ; X_{e}^{\prime} ; X_{e}^{\prime} \rightarrow X_{v}^{\prime}\right\}$ determines a graph of profinite groups $\mathcal{G}_{X}$ associated to the stable curve $X$.

When considering the case of a curve with marked points (i.e., $r>0$ ), it is useful to consider the following slightly modified "data with compact structure": Let us denote by $\Gamma_{X}^{\mathrm{c}}$ the semi-graph obtained from $\Gamma_{X}$ by appending to $\Gamma_{X}$, for each marked point $x \in \bar{X}$, the following:

an edge $e_{x} \stackrel{\text { def }}{=}\{x\}$, where $x \in e_{x}$ is sent by $\zeta_{e_{x}}$ to the vertex $v_{x}$ corresponding to the irreducible component of $\bar{X}$ that contains $x$.

We shall refer to the new edges " $e_{x}$ " that were added to $\Gamma_{X}$ to form $\Gamma_{X}^{\mathrm{c}}$ as the marked edges of $\Gamma_{X}^{c}$ and to $\Gamma_{X}^{c}$ itself as the dual graph with compact structure associated to $X$.

If, moreover, we associate to $e_{x}$ the scheme $X_{x}^{\prime}$ (which is noncanonically isomorphic to $\left.\operatorname{Spec}\left(k[[t]]\left[t^{-1}\right]\right)\right)$ obtained by removing $x$ from the completion of $\bar{X}$ at $x$, and apply " $\pi_{1}^{\text {tame }}(-)$ " to the natural morphism $X_{x}^{\prime} \rightarrow X_{v_{x}}^{\prime}$, then we obtain a natural semi-graph of profinite groups $\mathcal{G}_{X}^{\mathrm{c}}$ with underlying semi-graph $\Gamma_{X}^{\mathrm{c}}$. Moreover, one checks easily that when $k$ is of characteristic 0, the profinite group associated (as described above) to $\mathcal{G}_{X}$ or $\mathcal{G}_{X}^{\mathrm{c}}$ is isomorphic to " $\widehat{\Pi}_{g, r}$ ", i.e., the profinite completion of the fundamental group of a Riemann surface of genus $g$ with $r$ points removed.

In fact, if we take $k=\mathbb{C}$, and we think of the $X_{v}^{\prime}$ as Riemann surfaces and of the $X_{e}^{\prime}, X_{x}^{\prime}$ as "copies of the circle $\mathbb{S}^{1}$ ", then we see that this construction corresponds quite geometrically to gluing Riemann surfaces with boundary along copies of the circle. 
Finally, let us observe the following (valid for $k$ of arbitrary characteristic): The profinite group associated to $\mathcal{G}_{X}$ or $\mathcal{G}_{X}^{\mathrm{c}}$ may be identified with the admissible fundamental group

$$
\Pi_{X}^{\mathrm{adm}}
$$

of $(\bar{X}, D)$ (cf. [Mzk4], §2, 8; [Mzk3], §3). Moreover, if we define semi-graphs of groups $\mathcal{G}_{X}^{\text {et }}, \mathcal{G}_{X}^{\text {c,et }}$ by taking the profinite group at a vertex $v$ to be the tame fundamental group of $X_{v}$ [i.e., instead of $X_{v}^{\prime}$ ] and the profinite group at all of the edges to be trivial, then the resulting profinite group may be identified with the fundamental group

$$
\Pi_{X}^{\mathrm{et}}
$$

associated to the Galois category of finite étale coverings of $X$ which are tamely ramified at the cusps. In particular, we have a natural surjection $\Pi_{X}^{\mathrm{adm}} \rightarrow \Pi_{X}^{\mathrm{et}}$.

\section{Second Cohomology Groups:}

We continue with the notation of the above discussion. Let $l$ be a prime number distinct from the characteristic of $k$. In the following discussion, we shall use the notation " $H^{j}(-)$ " (respectively, " $H_{\mathrm{c}}^{j}(-)$ ") to denote the $j$-th étale cohomology module (respectively, $j$-th étale cohomology module with compact supports - cf. [Milne], Chapter III, Proposition 1.29; Remark 1.30) with coefficients in $\mathbb{Z}_{l}$.

If $v$ is a vertex of $\Gamma_{X}$, write $\bar{X}_{v}$ for the normalization of the corresponding irreducible component of $\bar{X}$, so $X_{v}^{\prime}=X^{\prime} \times_{\bar{X}} \bar{X}_{v}$. Then we have a natural "push-forward" isomorphism

$$
H_{\mathrm{c}}^{2}\left(X_{v}^{\prime}\right) \stackrel{\sim}{\rightarrow} H^{2}\left(\bar{X}_{v}\right)
$$

between free $\mathbb{Z}_{l}$-modules of rank 1 . Moreover, the natural morphisms $\bar{X}_{v} \rightarrow \bar{X}$ (as $v$ ranges over the vertices of $\Gamma_{X}$ ) determine natural restriction morphisms whose direct sum is easily verified to be an isomorphism:

$$
H^{2}(\bar{X}) \stackrel{\sim}{\rightarrow} \bigoplus_{v} H^{2}\left(\bar{X}_{v}\right)
$$

On the other hand, the composite of the natural "push-forward" morphism $H_{\mathrm{c}}^{2}\left(X_{v}^{\prime}\right) \rightarrow$ $H^{2}(\bar{X})$ with a restriction morphism $H^{2}(\bar{X}) \rightarrow H^{2}\left(\bar{X}_{w}\right)$, where $w \neq v$, is easily verified to be zero. [Indeed, this follows by considering the Chern class of the line bundle on $\bar{X}$ associated to a closed point of $X_{v}^{\prime}$ : This Chern class is the image of a generator of $H_{\mathrm{c}}^{2}\left(X_{v}^{\prime}\right)$ and, moreover, vanishes upon restriction to $\bar{X}_{w}$ since the restriction to $\bar{X}_{w}$ of this line bundle is clearly trivial.] In particular, we conclude that the direct sum of the natural "push-forward" morphisms $H_{\mathrm{c}}^{2}\left(X_{v}^{\prime}\right) \rightarrow H^{2}(\bar{X})$ yields an isomorphism

$$
\bigoplus_{v} H_{c}^{2}\left(X_{v}^{\prime}\right) \stackrel{\sim}{\rightarrow} H^{2}(\bar{X})
$$


which, if we identify $H_{\mathrm{c}}^{2}\left(X_{v}^{\prime}\right), H^{2}\left(\bar{X}_{v}\right)$ via the isomorphism $H_{\mathrm{c}}^{2}\left(X_{v}^{\prime}\right) \stackrel{\sim}{\rightarrow} H^{2}\left(\bar{X}_{v}\right)$, is inverse to the isomorphism $H^{2}(\bar{X}) \stackrel{\sim}{\rightarrow} \bigoplus_{v}, H^{2}\left(\bar{X}_{v}\right)$.

Put another way, if we identify the cohomology modules $H_{\mathrm{c}}^{2}\left(X_{v}^{\prime}\right), H^{2}\left(\bar{X}_{v}\right)$ via the isomorphism $H_{\mathrm{c}}^{2}\left(X_{v}^{\prime}\right) \stackrel{\sim}{\rightarrow} H^{2}\left(\bar{X}_{v}\right)$, then the natural "push-forward" morphism $H_{\mathrm{c}}^{2}\left(X_{v}^{\prime}\right) \rightarrow H^{2}(\bar{X})$ may be recovered as the composite

$$
H^{2}\left(\bar{X}_{v}\right) \hookrightarrow \bigoplus_{v} H^{2}\left(\bar{X}_{v}\right) \stackrel{\sim}{\rightarrow} H^{2}(\bar{X})
$$

of the natural inclusion of a direct summand with the inverse of the isomorphism $H^{2}(\bar{X}) \stackrel{\sim}{\rightarrow} \bigoplus_{v} H^{2}\left(\bar{X}_{v}\right)$ determined by the restriction morphisms.

Finally, let us observe that if $\bar{X}$ is sturdy (cf. [Mzk4], Definition 1.1) - i.e., every $\bar{X}_{v}$ has genus $\geq 2$ - then the natural morphisms from profinite group cohomology to étale cohomology give rise to a commutative diagram

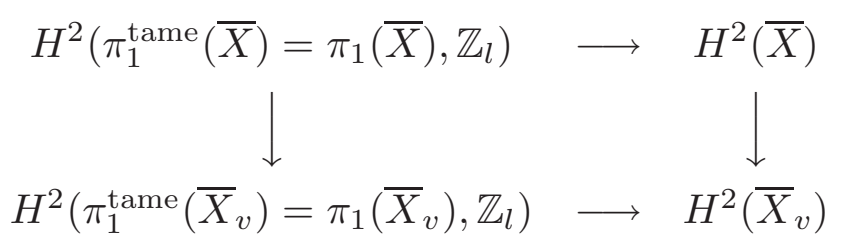

in which the horizontal morphisms are isomorphisms [since arbitrary étale cohomology classes with finite $l$-power torsion coefficients vanish upon restriction to some finite étale covering of $\bar{X}$ or $\bar{X}_{v}$ ].

\section{References}

[FC] G. Faltings and C.-L. Chai, Degenerations of Abelian Varieties, Springer-Verlag (1990).

[FJ] M. Fried and M. Jarden, Field Arithmetic, Springer-Verlag (1986).

[HR] W. Herfort and L. Ribes, Torsion elements and centralizers in free products of profinite groups, J. Reine Angew. Math. 358 (1985), pp. 155-161.

[Kato1] K. Kato, Logarithmic Structures of Fontaine-Illusie, Proceedings of the First JAMI Conference, Johns Hopkins Univ. Press (1990), pp. 191-224.

[Kato2] K. Kato, Toric Singularities, Amer. J. Math. 116 (1994), pp. 1073-1099.

[Knud] F. F. Knudsen, The Projectivity of the Moduli Space of Stable Curves, II, Math. Scand. 52 (1983), 161-199.

[Mtmo] M. Matsumoto, Galois representations on profinite braid groups on curves, J. Reine Angew. Math. 474 (1996), pp. 169-219. 
[Milne] J. S. Milne, Étale Cohomology, Princeton Mathematical Series 33, Princeton University Press (1980).

[Mzk1] S. Mochizuki, A Theory of Ordinary p-adic Curves, Publ. of RIMS 32 (1996), pp. 957-1151.

[Mzk2] S. Mochizuki, Foundations of p-adic Teichmüller Theory, AMS/IP Studies in Advanced Mathematics 11, American Mathematical Society/International Press (1999).

[Mzk3] S. Mochizuki, The Geometry of the Compactification of the Hurwitz Scheme, Publ. of RIMS 31 (1995), pp. 355-441.

[Mzk4] S. Mochizuki, The Profinite Grothendieck Conjecture for Closed Hyperbolic Curves over Number Fields, J. Math. Sci. Univ. Tokyo 3 (1996), pp. 571-627.

[Mzk5] S. Mochizuki, A Version of the Grothendieck Conjecture for $p$-adic Local Fields, The International Journal of Math. 8 (1997), pp. 499-506.

[Mzk6] S. Mochizuki, The Local Pro-p Anabelian Geometry of Curves, Invent. Math. 138 (1999), pp. 319-423.

[Mumf] D. Mumford, Abelian Varieties, Oxford Univ. Press (1974).

[Naka1] H. Nakamura, Galois rigidity of pure sphere braid groups and profinite calculus, J. Math. Sci. Univ. Tokyo 1 (1994), pp. 71-136.

[Naka2] H. Nakamura, Galois rigidity of profinite fundamental groups [translation of Sugaku 47 (1995), pp. 1-17], Sugaku Expositions 10 (1997), pp. 195-215.

[NTM] H. Nakamura, A. Tamagawa, and S. Mochizuki, The Grothendieck Conjecture on the Fundamental Groups of Algebraic Curves, Sugaku Expositions 14 (2001), pp. $31-53$.

[NSW] J. Neukirch, A. Schmidt, K. Wingberg, Cohomology of number fields, Grundlehren der Mathematischen Wissenschaften 323, Springer-Verlag (2000).

[Serre1] J.-P. Serre, Trees, Springer-Verlag (1980).

[Serre2] J.-P. Serre, Local Class Field Theory in Algebraic Number Theory, ed. J.W.S. Cassels and A. Fröhlich, Academic Press (1967).

[SGA1] Revêtement étales et groupe fondamental, Séminaire de Géometrie Algébrique du Bois Marie 1960-1961 (SGA1), dirigé par A. Grothendieck, augmenté de deux exposés de M. Raynaud, Lecture Notes in Mathematics 224, Springer-Verlag (1971).

[Tama1] A. Tamagawa, The Grothendieck Conjecture for Affine Curves, Compositio Math. 109 (1997), pp. 135-194.

[Tama2] A. Tamagawa, On the Fundamental Groups of Curves over Algebraically Closed Fields of Characteristic > 0, International Mathematics Research Notices 16 (1999), pp. 853-873. 
[Tate] J. Tate, p-Divisible Groups, Proceedings of a Conference on Local Fields, Driebergen, Springer Verlag (1967), pp. 158-183.

Research Institute for Mathematical Sciences,

KYoto UNIVERSITY,

KYОTO 606-8502, JAPAN

E-mail address: motizuki@kurims.kyoto-u.ac.jp 